\title{
Charging of carbon thin films in scanning and phase-plate transmission electron microscopy
}

Hettler, Simon; Kano, Emi; Dries, Manuel; Gerthsen, Dagmar; Pfaffmann, Lukas; Bruns, Michael; Beleggia, Marco; Malac, Marek

\section{Published in:}

Ultramicroscopy

Link to article, DOI:

10.1016/j.ultramic.2017.09.009

Publication date:

2018

Document Version

Peer reviewed version

Link back to DTU Orbit

Citation (APA):

Hettler, S., Kano, E., Dries, M., Gerthsen, D., Pfaffmann, L., Bruns, M., Beleggia, M., \& Malac, M. (2018). Charging of carbon thin films in scanning and phase-plate transmission electron microscopy. Ultramicroscopy, 184, 252-266. https://doi.org/10.1016/j.ultramic.2017.09.009

\section{General rights}

Copyright and moral rights for the publications made accessible in the public portal are retained by the authors and/or other copyright owners and it is a condition of accessing publications that users recognise and abide by the legal requirements associated with these rights.

- Users may download and print one copy of any publication from the public portal for the purpose of private study or research.

- You may not further distribute the material or use it for any profit-making activity or commercial gain

- You may freely distribute the URL identifying the publication in the public portal 


\title{
Charging of carbon thin films in scanning and phase-plate transmission electron microscopy
}

\author{
Simon Hettler, ${ }^{\mathrm{a}, *}$, Emi Kano ${ }^{\mathrm{b}}$, Manuel Dries ${ }^{\mathrm{a}}$, Dagmar Gerthsen ${ }^{\mathrm{a}}$, Lukas Pfaffmann ${ }^{\mathrm{c}}$, Michael \\ Bruns $^{\mathrm{c}}$, Marco Beleggia ${ }^{\mathrm{d}}$, Marek Malac ${ }^{\mathrm{b}}$ \\ ${ }^{a}$ Laboratory for Electron Microscopy, Karlsruhe Institute of Technology, Engesserstrasse 7, 76131 Karlsruhe, Germany \\ ${ }^{b}$ National Institute for Nanotechnology and Deptartment of Physics, University of Alberta, 11421 Saskatchewan Drive, \\ Edmonton, T6G 2M9, Canada \\ ${ }^{c}$ Institute for Applied Materials - Energy Storage Systems, Karlsruhe Institute of Technology \\ Hermann-von-Helmholtz-Platz 1, 76344 Eggenstein-Leopoldshafen, Germany \\ ${ }^{d}$ Center for Electron Nanoscopy, Technical University of Denmark, DK-2800 Kgs. Lyngby, Denmark
}

\begin{abstract}
A systematic study on charging of carbon thin films under intense electron-beam irradiation was performed in a transmission electron microscope to identify the underlying physics for the functionality of hole-free phase plates. Thin amorphous carbon films fabricated by different deposition techniques and single-layer graphene were studied. Clean thin films at moderate temperatures show small negative charging while thin films kept at an elevated temperature are stable and not prone to beam-generated charging. The charging is attributed to electron-stimulated desorption (ESD) of chemisorbed water molecules from the thin-film surfaces and an accompanying change of work function. The ESD interpretation is supported by experimental results obtained by electron-energy loss spectroscopy, hole-free phase plate imaging, secondary electron detection and x-ray photoelectron spectroscopy as well as simulations of the electrostatic potential distribution. The described ESD-based model explains previous experimental findings and is of general interest to any phase-related technique in a transmission electron microscope.
\end{abstract}

Keywords: scanning transmission electron microscopy, transmission electron microscopy, electron-beam induced charging, thin film, phase plate, radiation damage, hole-free phase plate, Volta phase plate

\section{1. Introduction}

Charging of insulating specimens is a well-known issue in electron microscopy, including transmission (TEM), scanning TEM (STEM) and scanning electron microscopy (SEM) and can lead to severe image degradation and sample damage. The charging effect is pronounced in cryo (S)TEM and SEM applications because the electrical conductivity of typical biological samples is very low at liquid nitrogen temperature [1]. Although a thin layer of amorphous carbon $(\mathrm{aC})$ 7 is typically sufficient to reduce charging effects to an acceptable level, it turns out that $\mathrm{aC}$ films 8 are prone to charging themselves, commonly referred to as the "Berriman effect" [2]. This led

${ }^{*}$ Corresponding author: simon.hettler@kit.edu

Preprint submitted to Ultramicroscopy 
to a search for an optimal specimen support for cryo applications and other means of reducing specimen charging [3-5]. The origin of most charging effects is the generation of secondary electrons (SEs) which escape from the specimen and leave positively charged ions behind [68]. For insulating materials, the electrostatic charging remains even at elevated temperatures and affects the electron beam leading to severe image degradation. If the electrical conductivity of the specimen is sufficiently high, the charge is compensated by a current from ground and observable charging should not be present. However, charging effects were observed on aC, a material which is assumed to have sufficient electrical conductivity [2]. Proposed explanations are that the surface of the $\mathrm{aC}$ layer has insulating properties or that charging occurs in a contamination layer adsorbed on the aC film $[9,10]$.

Only recently, the charging of aC thin films has found a beneficial application in the use as hole-free phase plate (PP) for TEM [8]. PPs increase the image contrast of electron-transparent objects by inducing a phase shift between the unscattered and scattered part of the electron wave in the back focal plane (BFP) of the objective lens [11-13]. The charging of aC films is exploited by placing a uniform thin film in the BFP on which a charged area is created by the intense zero-order beam of unscattered electrons causing the desired phase shift. This concept of the hole-free (HF) PP has overcome several limitations of previous PP approaches [8]. Interestingly, both polarities of the charged area, positive and negative, have been reported in the literature $[8,14,15]$. While positive charging can be well explained by an insulating surface of the aC film, the physical reason for negative charging is still not understood.

In the present study, we systematically analyzed three $\mathrm{aC}$ thin films fabricated by different evaporation devices and a single-layer graphene (SLG) sample in a transmission electron microscope to identify the physical reasons for the charging of $C$ thin films. We performed electronenergy loss spectroscopy (EELS) and HFPP imaging at different thin-film temperatures yielding the trace of the film thickness and a measure for phase shift arising from a change in the film thickness and from possible charging of the thin film. To separate charging from contamination effects, different steps were undertaken to avoid contamination of the thin films [16]. X-ray photoelectron spectroscopy (XPS) data provides additional information on the chemical composition of the thin film surfaces. The experimental results indicate that charging of clean $\mathrm{C}$ thin films is small, has a negative polarity and its magnitude decreases with increasing temperature. The negative polarity of the charge is explained by a local change in the work function (WF) induced by electron-stimulated desorption (ESD) of chemisorbed molecules on the thin-film surface which is supported by simulations of the electrostatic potential. This effect also describes the functionality of HFPPs with negative polarity [14] and is of general interest to any phase-related technique in a transmission electron microscope.

\section{Materials and methods}

We used the identical methods to analyze the phase-plate films, referred to as thin films in the following, as in our recent publication [16]. TEM experiments were performed in a dry-pumped Hitachi HF-3300 transmission electron microscope (Hitachi High Technologies, Naka, Japan) with a cold field emission gun operated at $300 \mathrm{kV}$. The free-lens mode was used to set up the electron optics in order that the BFP of an additional test specimen placed in the condenser-lens system coincides with the standard object plane (Figure 1). For the collection of a time series of EELS spectra with a Gatan Image Filter (GIF Tridiem model 863, Gatan Inc., Pleasanton, CA, USA) the test specimen, a standard carbon replica sample with a 2160 lines/mm gold diffraction grating (Ted Pella, prod. no 607), was retracted to measure solely the electron-beam induced 
Figure 1: Schematic illustration of the electron-optical setup used to study charging of carbon thin films. An additional test specimen is implemented in an aperture holder above the objective lens. The tested phase-plate film is inserted in the sample holder plane that coincides with the BFP of the objective lens prefield. This allows to rapidly change the thin films and to heat the film or measure the return current to ground by using appropriate TEM holders. The imaging lenses can be adjusted for either EELS measurements or HFPP imaging. A SE detector is used to simultaneously record the SE time evolution with the EELS signal. Reprinted from [16] with permission.

EELS and HFPP data give information on changes of the thin-film thickness and on charging processes. The acquired low-loss (LL) EELS spectra allow the determination of the thickness evolution of the thin film by calculating

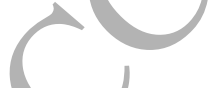

thickness change of the thin film. The time evolution of the SE emission current from the thin film is acquired simultaneously with the time evolution of EELS spectra. For acquisition of HFPP image series using a Gatan Ultrascan 1000 camera (Gatan Inc.), the test specimen is inserted. The

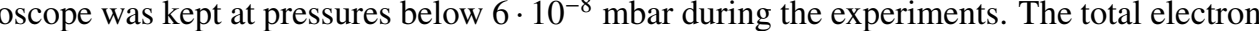
order of $\mathrm{L}=3 \mathrm{~mm}$ and the computer control system for the electron microscopy laboratory was used for data aequisition [17].

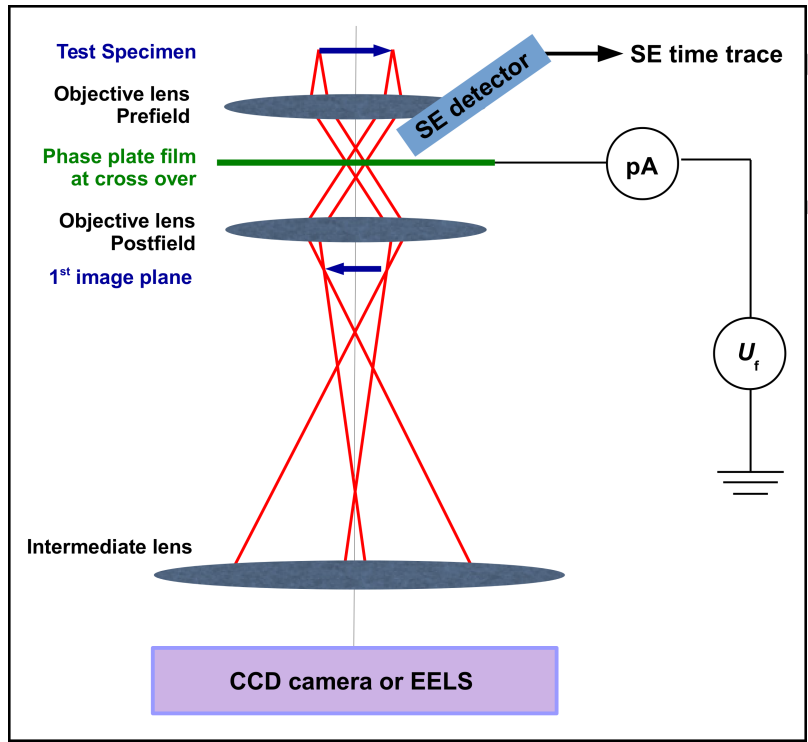

with the intensity of the zero-loss peak $I_{Z L}$, the intensity of the LL region $I_{L L}$, the thin-film thickness $t$ and the inelastic mean free path $\lambda[18]$.

${ }_{68}$ The power spectrum of a HFPP TEM image contains information on a phase shift caused 69 by an electron-beam induced modification of the thin film. The Thon rings in power spectra of 70 phase-contrast TEM images can be described by the phase-contrast transfer function (PCTF).

71 Due to the low magnification achievable in this electron-optical setup, strong overfocus (-) val72 ues of $\Delta f$ between 100 and $500 \mu \mathrm{m}$ were used to ensure a good visibility of Thon-rings. The 
intermediate lens is used for defocusing. The effect of spherical aberration can be neglected for the high defocus values leading to the PCTF [19]

$$
P C T F \propto \sin \left(-\pi \cdot \Delta f \cdot \lambda_{e} \cdot q^{2}+\phi_{P P}\right)
$$

with the electron wavelength $\lambda_{e}$ and the spatial frequency $q . \phi_{P P}$ is the phase shift of the unscattered electrons $(q=0)$ relative to the scattered electrons $(q>0)$ caused by charging of the thin film. In this convention, a positively charged patch on the thin film implies a positive value for $\phi_{P P}$. Detailed information on the determination of the charge polarity can be found in [15].

The phase shift $\phi_{P P}$ and defocus $\Delta f$ of the images are determined by a Matlab-based (MathWorks, Natick, MA, USA) pattern recognition software [20] under the assumption that the phase shift is only induced on the unscattered electrons. A time series of HFPP TEM images allows to trace the PCTF evolution and thus the phase shift induced by an electron-beam induced modification of the thin film. As the PCTF appears squared in the power spectra, a phase shift of $\pi$ between two power spectra cannot be distinguished. This implies that the sign of phase shift steps between subsequent power spectra of close to $\pi / 2$ is ambiguous.

The XPS analysis was performed using a K-Alpha ${ }^{+}$spectrometer (Thermo Fisher Scientific, Waltham, MA, USA) equipped with an $\mathrm{Al}-\mathrm{K}_{\alpha}$ source $(1486.6 \mathrm{eV})$ and a $400 \mu \mathrm{m}$ sized X-Ray probe. Surface sputtering with $8 \mathrm{keV}$ Ar clusters (2000 atoms, $4 \mathrm{eV}$ per atom) was carried out to clean the thin films from contamination and to study the actual thin-film properties. An electron flood gun was used to compensate charging of the thin film during the measurement. Data analysis and quantification of the bond structures was performed using the Avantage software (Thermo Fisher Scientific).

Table 1 gives an overview over the investigated $C$ thin films and their labeling within this article. The PVD, EB and Thread aC thin films were fabricated by evaporation of a thin layer on a freshly cleaved mica substrate (Plano, Wetzlar, Germany; Part No. 54) and were transfered to $\mathrm{Cu}$ grids (Plano; Part No. G2150C/G2300C) in a subsequent floating process. Electron-beam evaporation (EBE) was used to deposit the PVD aC and EB aC thin films using the PVD 75 (Kurt J. Lesker Company, Hastings, UK) for the PVD aC thin film. The EB aC thin film was provided by Michael Marko (Wadsworth Centre, New York State University, Albany, NY, USA) and was fabricated with the Leica MED020 (Leica Microsystems, Wetzlar, Germany). The Thread aC thin film was evaporated using a custom-built carbon-thread evaporation system. The thickness of the PVD and Thread aC thin film was measured by TEM analysis of a cross-section TEM specimen prepared by focused-ion-beam milling from a simultaneously coated Si wafer. The thickness of the EB aC thin film was measured during the evaporation process with a crystal thickness monitor. The thin films on their supporting grids were kept in clean plastic boxes for a longer time period prior to transfer into the electron microscope. The single-layer graphene (SLG) sample was grown on a $\mathrm{Cu}$ foil by chemical vapor deposition and then transferred to TEM grids using a previously described method [21]. We used gold TEM grids covered with an amorphous carbon film with $2 \mu \mathrm{m}$ diameter holes (Quantifoil, Quantifoil Micro Tools GmbH, Jena, Germany).

To avoid contamination, the thin films were cleaned by different methods as noted in Table 1 . UV cleaning [22] was carried out ex-situ using a 10 min cleaning procedure in the ZONE Cleaner (Hitachi High Technologies Canada) for each side of the thin film. Heating of the thin films was performed in situ and overnight at a temperature of $>250{ }^{\circ} \mathrm{C}$ using a Gatan heating holder (Gatan Inc.). Beam shower was performed by illuminating a large area around the region of interest with a spread $300 \mathrm{keV}$ beam with total areal doses $<0.15 \frac{\mathrm{C}}{\mathrm{cm}^{2}}$. A Ronchigram was utilized to ensure 


\begin{tabular}{|c|c|c|c|}
\hline Label & Preparation & Thickness & Cleaning procedure \\
\hline PVD aC & EBE in Lesker PVD 75 & $10 \mathrm{~nm}$ & UV / heating \\
EB aC & EBE in Leica MED20 & $12 \mathrm{~nm}$ & heating \\
Thread aC & carbon-thread evaporation & $5-7 \mathrm{~nm}$ & heating \\
SLG & Single-layer graphene & - & beam shower / heating \\
\hline
\end{tabular}

Table 1: List of analyzed C thin films.

that the BFP coincides with the thin film [23]. Multiple data collection runs were performed on each thin film and experimental condition, such as the thin-film temperature. Each experimental run was performed on a fresh area of the thin film.

\section{Experimental Results}

In this section we present the experimental results on thickness, SE and phase shift evolution obtained by EELS and HFPP imaging. Subsequently, we show XPS measurements performed on the PVD aC and $\mathrm{EB} \mathrm{aC}$ thin films before presenting an analysis of previously published HFPP image series.

\subsection{Evolution of the relative thickness}

Tracing the relative thickness $(t / \lambda)$ evolution reveals processes linked to a deposition or removal of material. Figure 2 shows exemplary $\mathrm{t} / \lambda$ evolutions for the three $\mathrm{aC}$ and the SLG thin films in dependence of the areal electron dose. The time axes at the top of Figure 2 and in the following Figures are calculated for an electron beam current of $60 \mathrm{pA}$ and is for illustration purposes only. The corresponding TEM images of the thin films acquired at a strong overfocus $(<-1 \mathrm{~mm})$ after the experimental series are displayed in Figure 3. The Thread aC thin film was heated in situ for 2 nights at $275^{\circ} \mathrm{C}$ to inhibit contamination and the PVD aC was cleaned in a UV cleaning step prior to inserting it in the microscope. After either of the two cleaning procedures, the thin films show a flat $t / \lambda$ evolution indicating that there is neither hole-drilling nor contamination (solid black and blue lines in Figure 2a). If the thin film is kept at room temperature (RT) in the microscope for a few hours, contamination can recur as observable by the increasing $t / \lambda$ curve for the Thread aC thin film (dashed blue line in Figure 2a). While the electron beam does not leave a visible mark on the UV cleaned PVD aC at RT and the Thread aC thin film at $300^{\circ} \mathrm{C}$ (Figure $3 \mathrm{e}$ and $\mathrm{f}$ ), the contamination spot is clearly visible as a bright spot in Figure $3 \mathrm{~g}$ for the Thread aC kept at RT for $2.5 \mathrm{~h}$ due to the strong overfocus. Slight mechanical drift of the thin film during data acquisition can be recognized as a minor elongation of the spot.

A different behavior is observed for the $\mathrm{EB} \mathrm{aC}$ thin film. If the thin film is inserted in the microscope without prior cleaning step and directly heated to $260{ }^{\circ} \mathrm{C}$, the $t / \lambda$ curve shows a strong initial decrease followed by a slower increase (solid red line in Figure 2b). A TEM image taken directly after the initial decrease reveals a slightly bright area surrounded by a darker ring and a bright halo (Figure 3c) whereas the image taken at the end of the measurement reveals a strong bright spot with dark halo indicating the deposition of contamination. Heating the $\mathrm{EB} \mathrm{aC}$ thin film in situ at $260^{\circ} \mathrm{C}$ overnight leads to a strong decrease in the initial $t / \lambda$ value from $\approx$ 0.2 on the first day (solid red line) to 0.08 on the 2 nd day (dotted red line) followed by a minor decrease to 0.07 upon electron-beam illumination. Another two nights of in-situ heating do not further decrease $t / \lambda$ but yield an almost flat $t / \lambda$ evolution (dashed red line). 


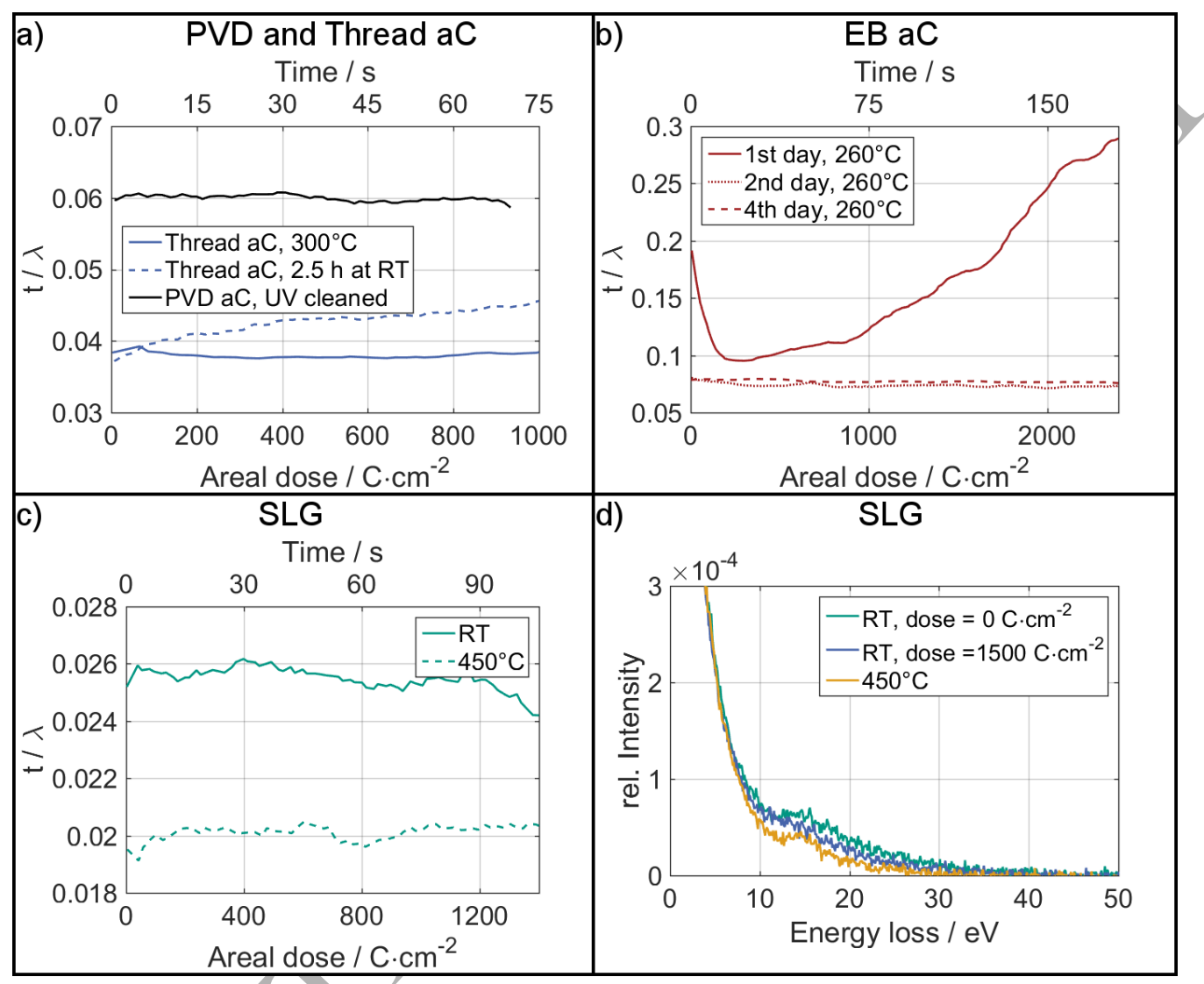

Figure 2: Exemplary $t / \lambda$ evolutions in dependence of the areal dose for the investigated $C$ thin films and EELS analysis of the SLG thin film. (a) Both PVD and Thread aC show a flat $t / \lambda$ evolution after the respective cleaning procedures (solid black and blue lines). Leaving the Thread aC thin film at RT for $2.5 \mathrm{~h}$ leads to the recurrence of contamination as visible by an increasing $t / \lambda$ (dashed blue). (b) The EB aC thin film shows a strong initial decrease followed by a slower increase if the thin film is heated directly after insertion in the microscope (solid red line). $t / \lambda$ is strongly decreased after overnight pumping at $260^{\circ} \mathrm{C}$ and only shows a minor decrease (dotted red). After 3 days of in-situ heating, the $t / \lambda$ evolution is almost flat (dashed red). (c) The SLG thin film exhibits a lower $t / \lambda$ value after heating to $450{ }^{\circ} \mathrm{C}$ overnight (dashed green line) compared to the value measured at RT (solid green). The evolution is flat at elevated temperature and shows a decrease at RT. (d) The EELS spectra of the SLG thin film acquired before (green line) and after (blue) the RT measurement depicted by the blue line in (c) show the decrease in thickness. The EELS spectra acquired at $450{ }^{\circ} \mathrm{C}$ shows an overall decreased intensity and exhibits a peak at $\approx 15 \mathrm{eV}$ (yellow line). The time axes is calculated for an electron beam current of $60 \mathrm{pA}$ and is for illustration purposes only. (For interpretation of the references to color in this figure legend, the reader is referred to the web version of the article.) 


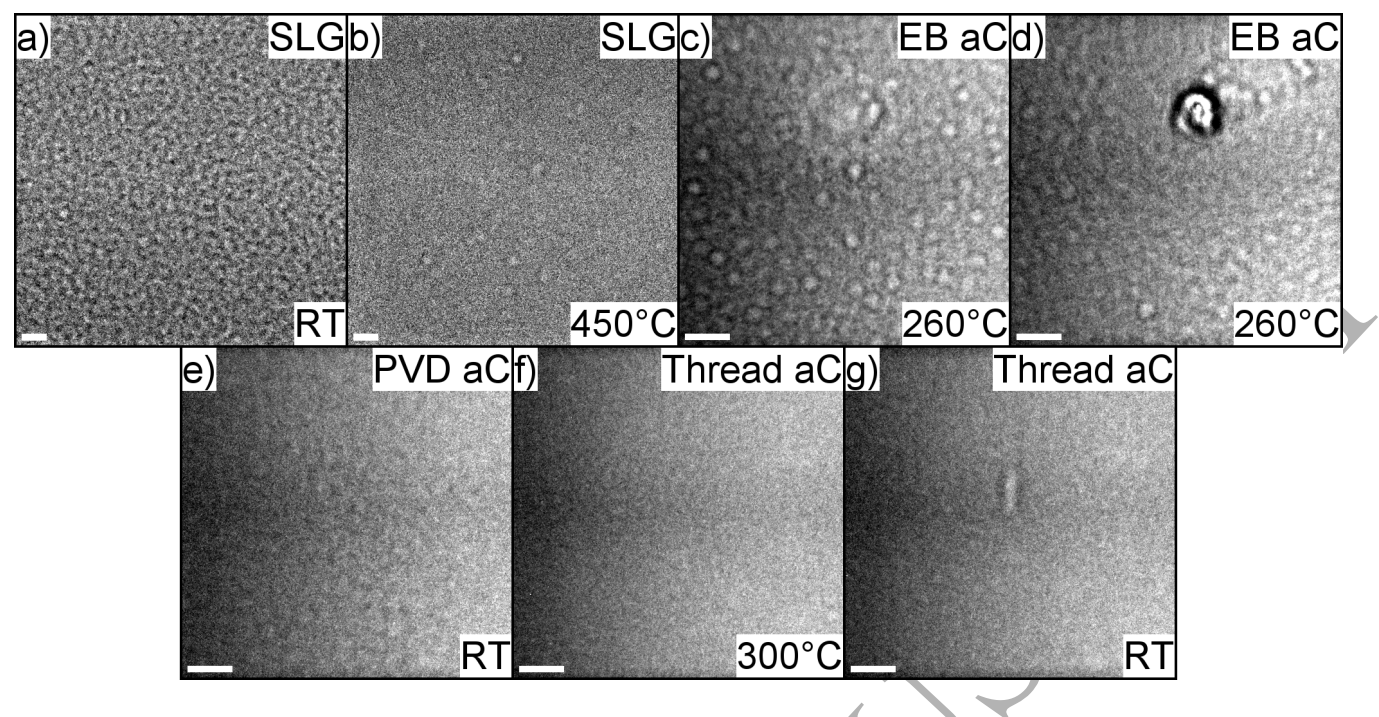

Figure 3: TEM images acquired at strong overfocus $(<-1 \mathrm{~mm})$ corresponding to the $t / \lambda$ measurements displayed in Figure 2 for the SLG thin film at (a) RT and (b) $450{ }^{\circ} \mathrm{C}$, the $\mathrm{EB} \mathrm{aC}$ thin film at $260{ }^{\circ} \mathrm{C}$ (c) after the initial decrease and (d) after the complete $t / \lambda$ measurement, (e) the PVD aC at RT and the Thread aC thin film at (f) $300{ }^{\circ} \mathrm{C}$ and (g) RT. The scale bars have a size of $50 \mathrm{~nm}$.

Decreasing $t / \lambda$ values are also observed fór the SLG thin film at RT (solid green line in Figure 2c) while a flat evolution is observed at $450^{\circ} \mathrm{C}$ (dashed green line). To avoid contamination and instabilities of the SLG, a beam shower with a dose between 0.1 and $0.15 \frac{\mathrm{C}}{\mathrm{cm}^{2}}$ was carried out directly before the measurements. The lower $t / \lambda$ value observed at $450{ }^{\circ} \mathrm{C}$ and the difference in TEM image contrast between the SLG thin film at RT (Figure 3a) and at $450{ }^{\circ} \mathrm{C}$ (Figure 3b) show that there is a thin amorphous layer adsorbed on the SLG at RT. A comparison of the EELS spectra acquired at the beginning (green line in Figure 2d) and at the end (yellow line) of the measurement series at RT corresponding to the $t / \lambda$ data in Figure $2 \mathrm{c}$ suggests that parts of the amorphous layer are removed during electron-beam illumination although a distinct mark of the electron beam is not visible in a TEM image (Figure 3a). While the plasmon peak at $15 \mathrm{eV}$ is clearly visible for the clean $S L G$ at $450{ }^{\circ} \mathrm{C}$ (yellow line in Figure 2d), the peak appears blurred at RT, possibly induced by the amorphous layer on the surface (green and blue lines) [24].

\subsection{SE evolution}

The time evolution of the SE signal gives an insight into the rate at which the SEs escape from the thin film and can provide information on topographic changes or electric potentials on the surface. Figure 4 shows the SE traces for the thin films acquired simultaneously with the $t / \lambda$ curves displayed in Figure 2. A comparison between the PVD (black line) and Thread $\mathrm{aC}$ (blue line) thin film is shown in Figure 4a. The PVD aC thin film exhibits a higher and slowly decreasing SE signal while the SE signal is weaker and almost flat for the Thread aC thin film. For thin films with thicknesses in the range of the SE escape depth, a change of the film thickness can influence the SE yield [16]. As both thin films don't show a significant change in $t / \lambda$ (Figure 2a), the decreasing SE signal of the PVD aC thin film is likely to result from a charging process. 


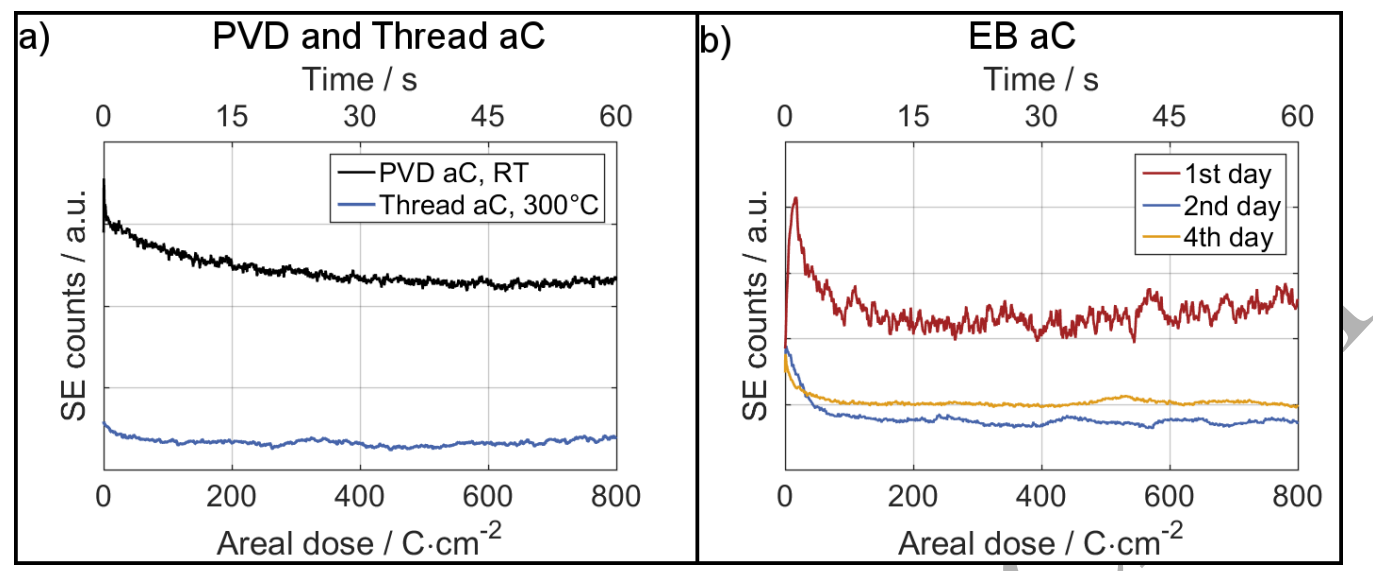

Figure 4: SE evolution depending on the areal dose simultaneously acquired with the $t / \lambda$ data (Figure 2) for (a) the PVD and Thread $\mathrm{aC}$ and (b) the $\mathrm{EB} \mathrm{aC}$. The time axes is calculated for an electron beam current of $60 \mathrm{pA}$ and is for illustration purposes only. (For interpretation of the references to color in this figure legend, the reader is referred to the web version of the article.)

Three SE curves for the $\mathrm{EB}$ aC thin film at $260^{\circ} \mathrm{C}$ on the first (red line), second (blue line) and fourth day (yellow line) in the microscope are depicted in Figure 4b. A strong increase in the SE signal is observed on the first day which is followed by a decrease before the signal slowly starts to rise again (red line). On the second and fourth day, the signal is reduced and initially shows a minor decrease before flattening. Except for the sharp increase on the first day, all curves show the similar shape as the $t / \lambda$ evolution (Figure $2 \mathrm{~b}$ ). Almost no change in the SE signal is observed for the SLG thin film (supplementary data). The SE trace acquired at RT shows a small decrease after an areal dose of $400 \frac{\mathrm{C}}{\mathrm{cm}^{2}}$ while the trace acquired at $450{ }^{\circ} \mathrm{C}$ is flat.

\subsection{PCTF evolution}

The acquisition of HFPP images in short time intervals and the subsequent analysis of the corresponding power spectra allows to trace the evolution of the PCTF and thus the phase shift induced by an electron-beaminduced modification of the illuminated area on the thin film. The PCTF analysis of the HFPP image series acquired for the SLG thin film at RT is shown in Figure 5a by the comparison of two PCTFs from the beginning (solid green line) and the end (dashed green line) of the respective series. In Figure 5a a shift between the initial (solid green line) and the final (dashed green line) PCTF can be observed. The shift of minima and maxima towards higher spatial frequencies (dashed green line) indicates that an additional negative phase shift is induced on the unscattered electrons for the HFPP image acquired at the end of the series. The amount of phase shift is determined to $\phi_{P P} \approx-0.2 \pi$ and can be attributed to negative charging of the irradiated area on the SLG thin film. The shift continuously increases for spatial frequencies below $0.02 \mathrm{~nm}^{-1}$ but is constant for higher spatial frequencies. This suggests that the size of the phase shift tails caused by the charged area, which is not identical to the beam diameter, does not exceed $0.02 \mathrm{~nm}^{-1}$ corresponding to a real-space disc with a diameter of $2 \cdot L \cdot \lambda_{e} \cdot 0.02 \mathrm{~nm}^{-1} \approx 250 \mathrm{~nm}$ with the camera length $\mathrm{L} \approx 3 \mathrm{~mm}$.

The results for the PVD aC, which was UV cleaned ex situ and analyzed at RT, are depicted in Figure 5b. The evolution of the PS determined from the power spectra of the HFPP images shows 

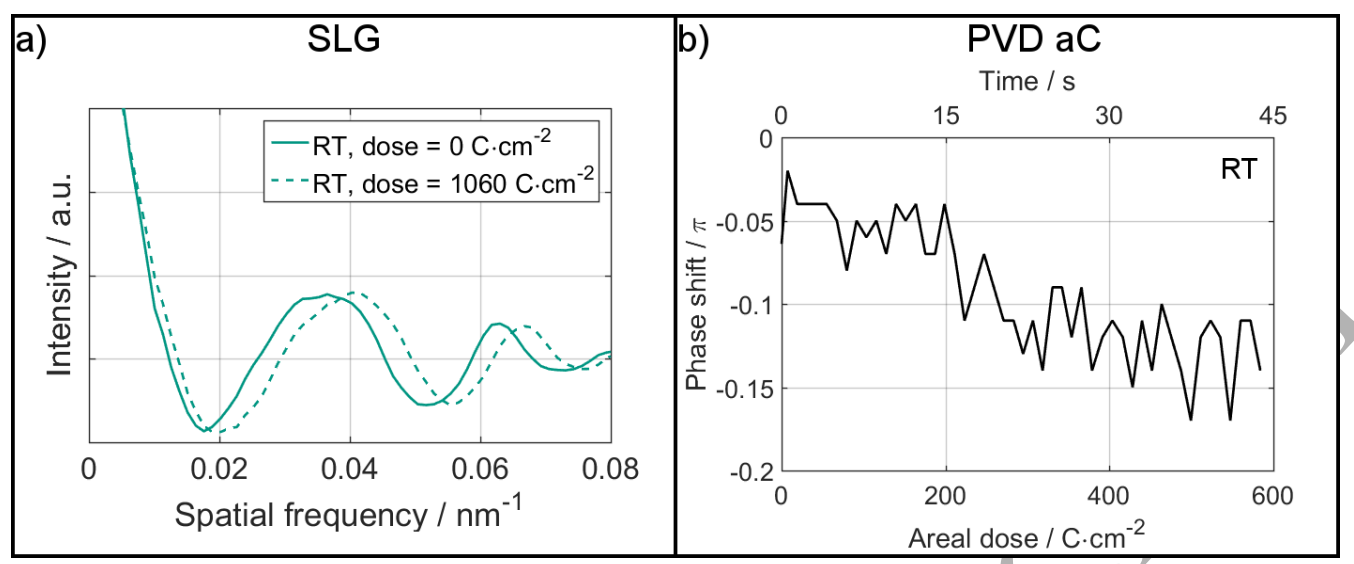

Figure 5: PCTF analysis of the SLG and the PVD aC thin films at RT. (a) Comparison of the initial (solid green line) and the final PCTF (dashed green line) of the HFPP image series shows a strong shift of Thon rings towards higher spatial frequencies. (b) Phase shift evolution for the UV cleaned PVD aC thin film at RT directly after insertion in the microscope shows a small negative phase shift. The time axes is calculated for an electron beam current of $60 \mathrm{pA}$ and is for illustration purposes only. (For interpretation of the references to color in this figure legend, the reader is referred to the web version of the article.)

the buildup of a small negative phase shift of $\approx-0.15 \pi$ which can be attributed to a negatively charged area on the thin film. If the SLG and PVD or Thread aC thin films are heated overnight at $450{ }^{\circ} \mathrm{C}$ and $275{ }^{\circ} \mathrm{C}$ in the microscope respectively, no phase shift or change in the PCTF is measured (supplementary data). This reveals that the heated SLG, PVD aC and Thread aC thin films are stable upon focused electron-beam illumination up to areal doses of $1200 \frac{\mathrm{C}}{\mathrm{cm}^{2}}$ and are not prone to neither positive nor negative charging.

a)

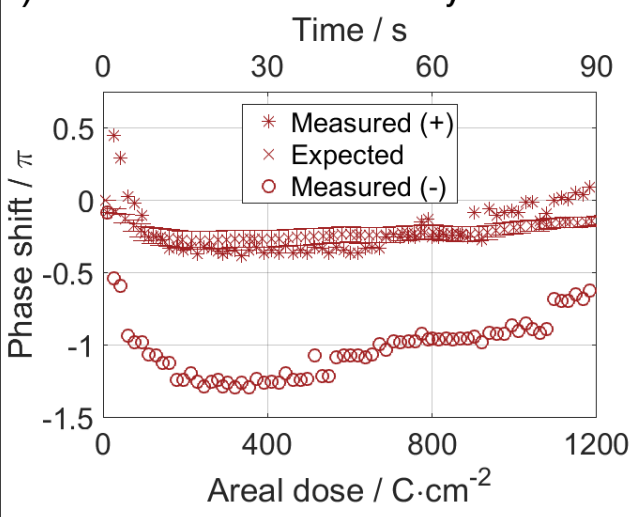

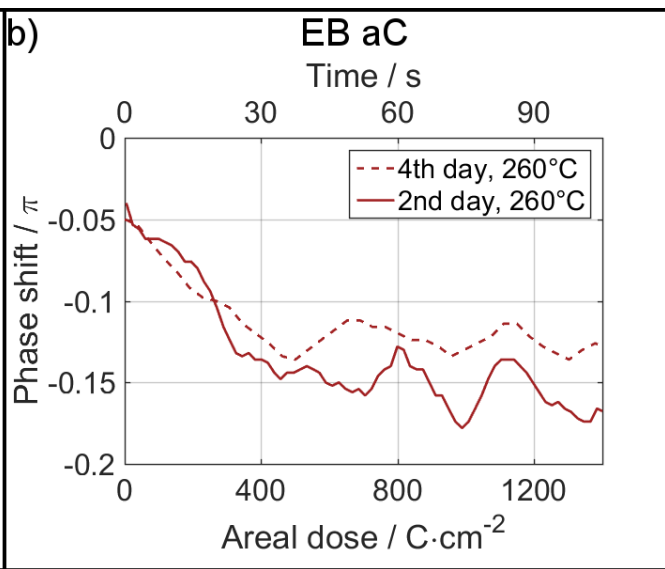

Figure 6: PCTF analysis of the EB aC thin film. (a) The measured phase shift evolution determined from the acquired HFPP images can be interpreted as initially positive (asterisk) or negative (circles) and is compared with the phase shift

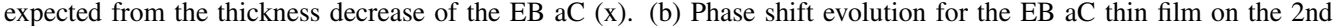
(solid red) and 4th day (dashed red) of heating to $260{ }^{\circ} \mathrm{C}$ in the microscope shows a small negative phase shift. The time axes is calculated for an electron beam current of $60 \mathrm{pA}$ and is for illustration purposes only. (For interpretation of the references to color in this figure legend, the reader is referred to the web version of the article.) 
The analysis of the phase shifting behavior of the $\mathrm{EB}$ aC thin film is depicted in Figure 6. The determination of the phase shift step between two subsequent power spectra is possible only in the interval between $-0.5 \pi$ and $0.5 \pi$ because a shift of $\pi$ leads to the identical power spectrum. This implies that a measured phase shift between two power spectra of $0.5 \pi$ is ambiguous and could be as well interpreted as $-0.5 \pi$. In the case of the $\mathrm{EB}$ aC thin film at $260{ }^{\circ} \mathrm{C}$ directly after insertion in the microscope, we initially measure a phase shift step of close to $0.5 \pi$ which can be interpreted either as positive (red asterisks in Figure 6a) or negative charging (red circles). After the initial phase shift step, the phase shift evolution tends to negative charging followed by a slow increase for larger areal doses in both cases. The decrease in thickness obtained from the $t / \lambda$ measurements (Figure 2b) can be converted in an expected decrease in phase shift if the mean inner electrostatic potential (MIP) and the inelastic mean free path $\lambda$ (MFP) of the material are known [25]. The inelastic MFP can be estimated from the measured film thickness during evaporation and acquired EELS spectra to be $\lambda=150 \pm 10 \mathrm{~nm}$. The MIP is assumed to be $9 \pm 1 \mathrm{~V}$ which is a common value for $\mathrm{aC}$ thin films [16, 26, 27]. The red crosses in Figure 6a show the expected phase shift evolution with the error bars reflecting the uncertainty in the inelastic MFP and the MIP. A comparison of the measured and expected phase shift shows that an additional phase shift of up to $\approx-\pi$ must be present for the negative phase shift interpretation (red circles). In case of the positive phase shift interpretation, the measured (asterisk) and expected $(+)$ curves agree well except for the initial discrepancy which could be explained by positive charging. If the $\mathrm{EB}$ aC thin film is consecutively heated to $260{ }^{\circ} \mathrm{C}$, a small negative phase shift is observed after both one and 3 days in the microscope (Figure $6 \mathrm{~b}$ ). As the corresponding $t / \lambda$ evolution is almost flat (Figure 2b), the phase shift is supposed to originate from negative charging of the thin film.

\subsection{XPS measurements}

To further study the apparent differences between the PVD and EB aC thin films, XPS experiments were conducted. Table 2 shows the results obtained for both films with and without surface cleaning and the corresponding XPS spectra can be found in the supplementary data. The $\mathrm{C} 1 \mathrm{~s}$ line is composed out of several peaks which can be attributed to different $\mathrm{C}$ bonds [28-30]. The first component is found at $284.4 \mathrm{eV}$ and corresponds to $\mathrm{sp}^{2}$-hypridized $\mathrm{C}$ atoms while the second peak at $285.0 \mathrm{eV}$ can be attributed to $\mathrm{sp}^{3}$-hypridized carbon atoms and bonds between $\mathrm{C}$ and $\mathrm{H}$. Two more peaks of much smaller intensity at $\approx 286$ and $288 \mathrm{eV}$ can be attributed to a $\mathrm{CO}$ or $\mathrm{C}=\mathrm{O}$ contamination formed at the surface of the samples due to air exposure. The fith contribution at a binding energy of $\approx 290 \mathrm{eV}$ is linked to $\pi-\pi^{*}$ transitions.

The analysis of the PVD aC thin film reveals the composition of the carbon bonds for the uncleaned and cleaned thin film (Table 2). In both cases, the peak corresponding to the $\mathrm{sp}^{2}$ hybridized C-C bonds has the highest intensity and slightly increases from $\approx 60$ to $70 \%$ due to the cleaning process. The binding energy of the $\mathrm{sp}^{3}$ bonds in the thin film and possible $\mathrm{C}-\mathrm{H}$ bonds originating from contamination is identical and amounts to $\approx 27$ and $23 \%$ before and after the cleaning process, respectively. The same trend is observed for the bonds between $\mathrm{C}$ and $\mathrm{O}$ which decrease by a factor of $\approx 2$ as a result of cleaning. The fifth contribution to the $\mathrm{C}$ bonds can be attributed to $\pi-\pi^{*}$ transitions and amounts to $\approx 3 \%$ in both cases.

The $\mathrm{sp}^{2}$ bonds contribute only $17 \%$ to the overall $\mathrm{C}$ bonds of the uncleaned $\mathrm{EB}$ aC thin film while $\approx 68 \%$ can be attributed to $\mathrm{sp}^{3} \mathrm{C}-\mathrm{C}$ or $\mathrm{C}-\mathrm{H}$ bonds. Also the contribution from the $\mathrm{C}-\mathrm{O}$ and $\mathrm{C}=\mathrm{O}$ bonds is increased while the peak at $290 \mathrm{eV}$ is missing. Cleaning of the $\mathrm{EB}$ aC thin film results in a strong change of the peaks (supplementary data) which now more closely resemble the shape of the PVD aC thin film. This is also reflected by the ratio between $\mathrm{sp}^{2}$ bonds $(66 \%)$ 


\begin{tabular}{|c|c|c|c|c|c|}
\hline & \multicolumn{2}{|c|}{ PVD aC } & \multicolumn{2}{c|}{ EB aC } & binding \\
bond / \% & uncleaned & cleaned & uncleaned & cleaned & energy / eV \\
\hline C-sp & 60.5 & 69.8 & 17.0 & 65.9 & $284.4-284.5$ \\
C-sp 3 / C-H & 26.5 & 22.8 & 67.5 & 27.2 & 285 \\
$\mathrm{C}-\mathrm{O}$ & 6.8 & 3.0 & 9.1 & 3.7 & $286.7-286.8$ \\
$\mathrm{C}=\mathrm{O}$ & 3.1 & 1.6 & 6.4 & 3.2 & $288.5-288.6$ \\
$\mathrm{C} \pi$ & 3.1 & 2.8 & 0 & 0 & 290 \\
\hline $\mathrm{C}$ & 88.7 & 97.8 & 70.5 & 71.4 & $280-294$ \\
$\mathrm{O}$ & 10.2 & 1.8 & 21.3 & 13.6 & $527-537$ \\
$\mathrm{Cu}$ & 0 & 0.1 & 3.8 & 10.6 & $925-965$ \\
$\mathrm{Si}$ & 1.1 & 0.3 & 4.4 & 4.4 & $97-106$ \\
\hline
\end{tabular}

Table 2: Peak energies and percentages determined from the XPS measurements on the PVD aC and EB aC thin films displayed in the supplementary data. The relative percentage of the $\mathrm{C}$ bonds and the complete atomic composition is shown for uncleaned and cleaned thin films.

and $\mathrm{sp}^{3} / \mathrm{C}-\mathrm{H}$ bonds $(27 \%)$ which lies between the values of the uncleaned and cleaned PVD aC thin film. Also the content of $\mathrm{C}$ and $\mathrm{O}$ bonds has decreased while $\pi-\pi^{*}$ transitions still are not present in the cleaned $\mathrm{EB}$ aC thin film.

In addition to $\mathrm{C}$, additional elements were found which are listed in Table 2. The presence of $\mathrm{Si}(<5 \%)$ is attributed to contaminants while $\mathrm{Cu}$ originates from the $\mathrm{Cu}$ support grids of the thin films. The $\mathrm{Cu}$ signal is negligible for the PVD aC thin film but is a significant contribution for the $\mathrm{EB} \mathrm{aC}$ thin film, especially after the cleaning procedure $(>10 \%)$. The $\mathrm{O}$ signal strongly decreases due to the cleaning procedure corresponding well to the decreasing amount of $\mathrm{C}-\mathrm{O}$ and $\mathrm{C}=\mathrm{O}$ bonds. The remaining $\mathrm{O}$ can be linked to bonds with $\mathrm{Si}$ and $\mathrm{Cu}$.

The XPS data together with a residual gas analysis within the Hitachi HF3300 (supplementary S2) reveal the presence of different species on the $\mathrm{C}$ thin films and in the residual gas atmosphere. Clearly, $\mathrm{C}$ atoms are the main constituents of the $\mathrm{C}$ thin films but oxygen and hydrogen atoms are as well present, mainly as adsorbates on the thin-film surfaces. As C-H bonds cannot be distinguished from C-C bonds in XPS, the amount of hydrogen on and in the thin-film surfaces remains unclear. However, after a suitable cleaning procedure, only a small number of adsorbed molecules remain. In a transmission electron microscope, where water has the highest partial pressure, water molecules will as well be present on the surfaces of the specimen.

\subsection{Analysis of published data on HFPPs}

For a comparison of our experimental results with previous studies on aC charging and HFPP performance, we analyzed the HFPP image series from [14] (supplementary movies S1 and S2) and [31] (supplementary movie 1) to extract the phase shift induced by the HFPP during settling. Both groups used an aC thin film very similar to the $\mathrm{EB}$ aC analyzed in this work and worked at a thin film temperature of $60{ }^{\circ} \mathrm{C}$ and $225^{\circ} \mathrm{C}$ [14] and $260{ }^{\circ} \mathrm{C}$ [31]. The beam diameter on the thin film was $1 \mu \mathrm{m}$ and $25 \mathrm{~nm}$ for the series acquired at $225^{\circ} \mathrm{C}$ (red line in Figure $7 \mathrm{a}$ ) and 60 ${ }^{\circ} \mathrm{C}$ (blue line in Figure 7a), respectively. The beam diameter is specified to be between 20 and $100 \mathrm{~nm}$ for the HFPP image series from [31] whose analysis is depicted in Figure 7b.

Figure 7a shows the phase shift evolution for the HFPP operated at $225^{\circ} \mathrm{C}$ (red line) which starts at $\approx-0.25$ and increases up to a phase shift of almost $-\pi$ without reaching a plateau. If the thin film is kept at only $60^{\circ} \mathrm{C}$, the phase shift evolution differs. The phase shift also starts with an increasing negative phase shift but tends towards a positive phase shift already after a few 


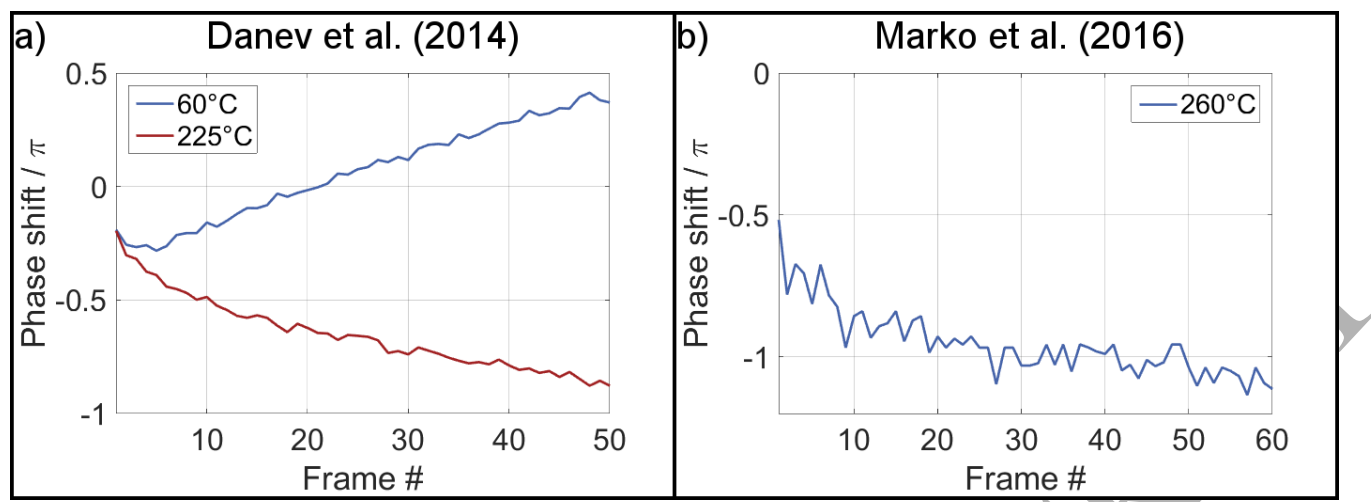

Figure 7: Determination of the PS of HFPP image series from (a) [14] and (b) [31].

frames followed by an almost linear increase (blue line in Figure 7a). The phase shift evolution determined from the HFPP image series acquired at $260{ }^{\circ} \mathrm{C}$ (Figure $7 \mathrm{~b}$ ) is similar to the data at $225^{\circ} \mathrm{C}$ (red line in Figure 7a): The phase shift initially starts at $-0.5 \pi$ and further increases to $-\pi$.

\section{Discussion}

In this section we discuss the experimental results and deduce the underlying microscopic phenomena responsible for the observed charging effects. We first assess the conditions under which charging occurs for the PVD aC, Thread aC and SLG thin films which show comparable charging characteristics. Electron-stimulated desorption (ESD) is suggested as the reason for the observed charging effects. A model of the charging phenomenon is established including the simulation of the electrostatic potential distribution followed by a discussion of the properties influencing the charging. The behavior of the $\mathrm{EB}$ aC thin film which deviates strongly from the other thin films is explained by structural differences. Finally, we discuss results from literature for which the presented model is a possible explanation and give an overview over the various HFPP implementations.

\subsection{Conditions for charging effects}

The assumption that thin films with reasonable conductivity, including $C$ thin films, do not charge under electron-beam illumination was disproved by Brink et al. [2] who observed charging of aC thin films in cryo TEM. Our experimental results, however, indicate that there are conditions under which $\mathrm{C}$ thin films do not show observable charging effects. Heating the Thread $\mathrm{aC}, \mathrm{PVD}$ aC or SLG thin films overnight to temperatures of $275^{\circ} \mathrm{C}$ and $450{ }^{\circ} \mathrm{C}$, respectively, leads to a thin film where no measurable thickness change or charging-induced phase shift can be observed (c.f. Figures 2a and c, supplementary data). As both PVD aC and SLG thin films exhibit charging if they are kept at RT (Figure 5) the increased temperature has to cause a modification of the thin films or their surfaces preventing their charging. As structural changes of the thin films such as crystallization are not observed in the conducted experiments, it seems likely that adsorbed molecules on the thin-film surfaces are a responsible cause of the charging effects. This implies that the PVD and Thread aC thin films themselves do not exhibit non-conductive 
surface sheets as suggested, e.g., in [9]. Even the Thread aC thin film with a thickness $<7 \mathrm{~nm}$ is stable up to electron doses of at least $1200 \mathrm{C} / \mathrm{cm}^{2}$, demonstrating that the thin film does not show any charging or thickness changes.

The fact, that the thin films have to be kept at an elevated temperature for a prolonged period of time to prevent charging, indicates that desorption processes are likely to be involved. Surface layers of adsorbates are present on the thin film shortly after insertion in the microscope. A common type of adorbates are hydrocarbon molecules which were identified as the source of contamination buildup in a transmission electron microscope [32]. In the following, the expression 'contamination' is used for cases, where a deposition of material is induced by the illuminating electron beam. Hydrocarbon molecules are physiosorbed on the surface with a low adsorption energy and are mobile on the thin-film surface. This is indicated by the fact that heating to $275{ }^{\circ} \mathrm{C}(\mathrm{kT} \approx 47 \mathrm{meV}$ ) overnight is sufficient to cause desorption of the hydrocarbon molecules [16]. The experimental results for the PVD aC and the SLG thin film conducted at RT, however, show the absence of contamination which can be inferred by the flat $t / \lambda$ evolution (Figure 2a and c). Contamination is inhibited by a removal of the hydrocarbon molecules in a previous UV cleaning step (PVD aC) and by beam shower (SLG) which immobilizes hydrocarbon molecules in an area much larger than the beam diameter [16]. Despite the absence of mobile hydrocarbon molecules on the thin film surface, negative charging effects are observed which are supposed to result from an additional, immobile, surface layer. The presence of such a surface layer, adsorbed with higher adsorption energy, was already assumed to be the reason for the measurement of similar diffusion coefficients for different thin-film materials [16]. As water is the most frequent molecule in the residual gas atmosphere of the Hitachi HF-3300 (supplementary data) and is known to easily adsorb on virtually any surface, it is reasonable to assume that $\mathrm{H}_{2} \mathrm{O}$ molecules are the dominant species forming the immobile surface layer.

In cases where charging was observed, the sign of the measured phase shift was negative for all thin films corresponding to a negatively charged area on the thin film. To understand the presence of a negative charge, the origin of phase shift induced on electrons during propagation through a thin film is reviewed. A homogeneous amorphous thin film is an object with a net charge of zero. Nevertheless, a positive phase shift is observed for transmitted electrons which is commonly explained by the MIP [25]. The physical reason is the difference in spatial distribution of positive and negative charges within the thin film. The positive charge at atomic nuclei is highly localized while the electrons are delocalized, especially the conduction band electrons. Fast electrons propagating through the film gain a phase shift mainly caused by the localized positive charges which are only partially screened by the core electrons in the thin film. The polarity of charge which is more localized determines the sign of the induced phase shift. The observation of negative charging on the $\mathrm{C}$ thin films therefore has to be caused by a more localized negative charge than positive charge within the illuminated area compared to the surrounding thin film. In contrast to suggesting the presence of additional, more localized, electrons in the illuminated area, we attribute the negative charging to a removal of adsorbed water molecules from the thin film surface. The process causing the removal of adsorbed water molecules and the resulting charging effects are discussed in the following sections.

\subsection{Electron-stimulated desorption can cause negative charging}

In the previous section we claimed that the removal of water molecules from the thin-film surfaces is responsible for the negative charging of the thin films. Here we propose that the underlying physical process for removal of adsorbed water is electron-stimulated desoprtion (ESD). ESD was intensively studied in the last century for numerous surfaces and molecules, 
atoms or molecular fragments [33, 34]. In the corresponding experiments, low-energy electrons $(<500 \mathrm{eV})$ were used to induce the desorption of adsorbates which were then analyzed using mass spectrometry. The threshold energy for ejecting ions or neutral molecules is in the range of $5-20 \mathrm{eV}$ with neutral species having a lower threshold [33]. In (S)TEM, the incident electron energy of $300 \mathrm{keV}$ is significantly higher than in typical ESD experiments resulting in a drastically decreased cross section for primary electrons to cause ESD. However, SEs generated in the thin film have typical kinetic energies of up to $100 \mathrm{eV}$ and thus can induce ESD. ESD indeed plays an important role in focused electron-beam induced processing (FEBIP) where electronbeam illumination is used in combination with suitable precursor gases to, e.g., deposit or etch nm sized structures on sample surfaces [35-37].

The principle of ESD is schematically depicted in Figure 8a: An illuminating primary electron (PE) generates SEs within the $\mathrm{C}$ thin film. The SEs can leave the thin film if their kinetic energy is larger than the work function of the material and can then be detected by a suitable detection system. During propagation through the thin film, the SE can initiate additional reactions including the desorption of adsorbed molecules from the thin-film surface. The probability for a PE to induce ESD is then dependent on the SE yield y, which was determined by measuring the total incident current and the return current from ground to be $y=0.001-0.01$ for the investigated thin films, multiplied by the probability of a SE to initiate the desorption of a water molecule. Additionally, the spatial and energy distribution of the generated SEs and the activation energy needed to cause the desorption of the adsorbed molecule play a role. With the areal PE current density of $\approx 1.6 \cdot 10^{7} \mathrm{e}^{-} /\left(\mathrm{nm}^{2} \mathrm{~s}\right)$ used in the experiment and a conservative estimate that only one of ten thousand generated SEs induces the desorption of an adsorbed molecule, the PE-induced desorption rate results in 2-16 molecules/ $\left(\mathrm{nm}^{2} \mathrm{~s}\right)$ depending on the SE yield of the thin film. This estimated cross-section is high enough to be considered as a possible cause for the observed charging phenomenon.

However, the removal of material by ESD and the associated thickness decrease alone cannot explain the observed charging phenomena. Both the PVD aC and SLG thin films show a flat or only slightly decreasing $t / \lambda$ evolution (Figure $2 \mathrm{a}$ and $\mathrm{c}$ ) suggesting that the desorbed molecules represent only a very small fraction of the actual film thickness. On the other hand, the negative phase shift is clearly detectable (Figure 5) showing that an additional effect has to be present to cause a negative charging of the illuminated area. This negative charging can be attributed to a local change in work function (WF) in the illuminated area which is caused by ESD. Studies on the effect of ad- or desorption of water molecules on clean metal surfaces revealed that the WF of the metal decreases if water is adsorbed on its surface [38-42]. The water molecule on a surface can be interpreted as a dipole with the positive charge pointing towards the vacuum [41] as schematically depicted in Figure $8 \mathrm{~b}$. This dipole layer represents a potential step $\delta \mathrm{V}$ which leads to an effectively decreasing WF of the metal and can amount up to a few eV depending on the properties of the metal. Although aC thin films are neither metallic nor crystalline, an adsorbed water layer with the hydrogen atom pointing to the vacuum will have the same effect on the WF of the investigated aC and SLG thin films. The effect on the WF is shown in Figure 9b: The WF in the central, 'dry', disk with radius $\mathrm{R}$ is equal to the nominal WF of $\mathrm{C}, \phi_{C}$, while it is decreased in the 'wet' areas by the adsorbed water to $\phi_{1}=\phi_{C}-\mathrm{e} \cdot \delta \mathrm{V}$.

The measurements of the WF change upon water adsorption showed an increasing $\delta \mathrm{V}$ with increasing water coverage which saturates once a monolayer of water is adsorbed on the surface [41]. The studies were carried out under ultra-high vacuum $\left(10^{-10} \mathrm{mbar}\right)$ and temperatures of liquid nitrogen $(77 \mathrm{~K})$. Compared to the conditions in a transmission electron microscope, the pressure is significantly reduced by more than 2 orders of magnitude and the temperature is 


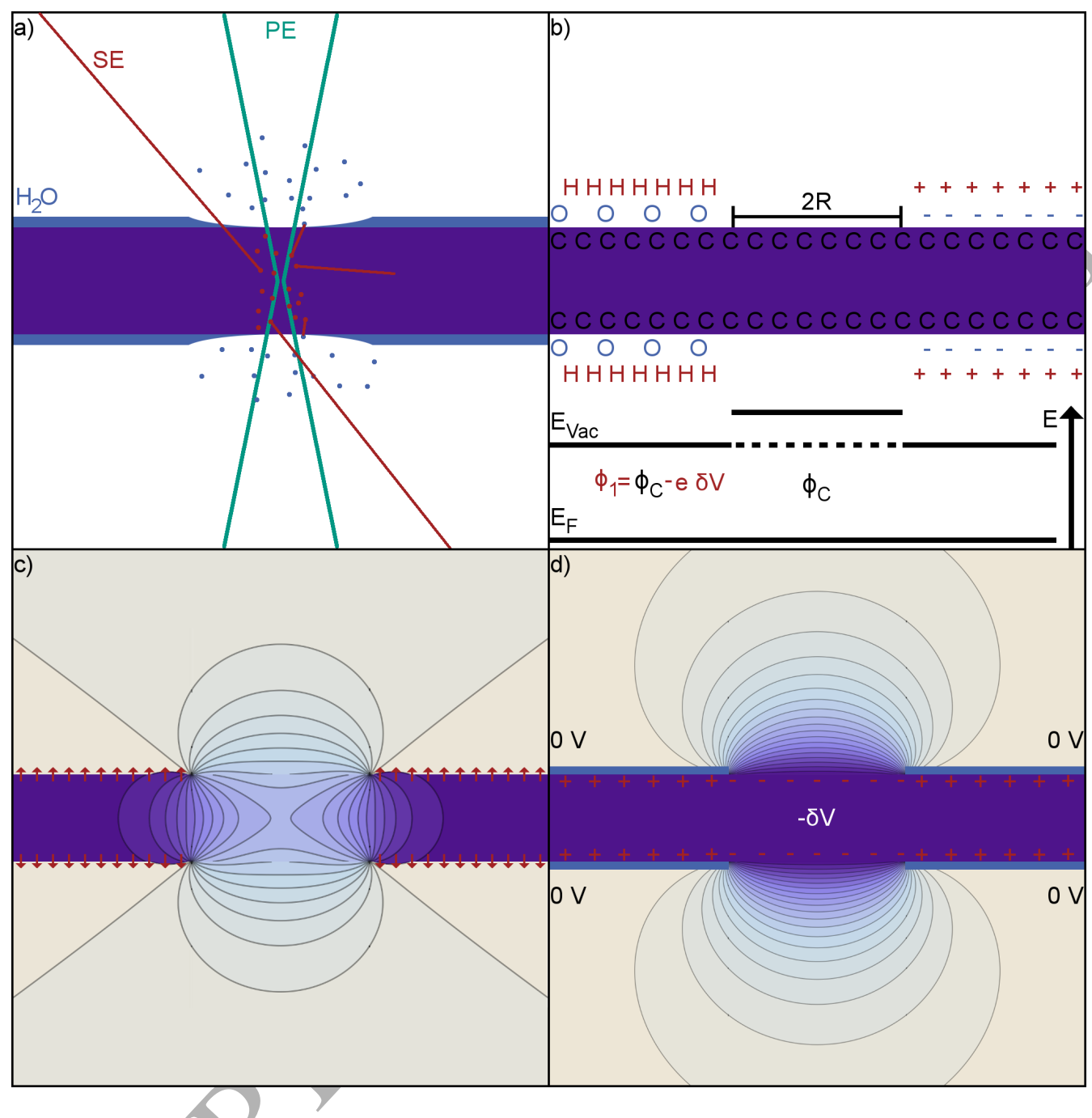

Figure 8: Schematic illustration of the principle of electron-stimulated desorption and its effect on the charge and potential distribution. (a) An illuminating primary electron (PE, green) is generating SEs (red) in the C thin film (purple) with a few exemplary SE trajectories indicated. A part of the SEs can initiate the desorption of adsorbed $\mathrm{H}_{2} \mathrm{O}$ molecules from a surface layer (blue). (b) The adsorbed water layer forms a dipole with the hydrogen (+) end pointing to the vacuum. The water layer effectively reduces the WF $\phi$, i.e., the energy difference between vacuum $\mathrm{E}_{\mathrm{Vac}}$ and Fermi-energy $\mathrm{E}_{\mathrm{F}}$ by e $\delta \mathrm{V}$. (c) Electrostatic potential distribution for two interrupted dipole layers (red arrows) in empty space without carbon film. The potential range is between the step $-\delta \mathrm{V}$ (purple) caused by the dipole layer and $0 \mathrm{~V}$ (white) (d) Potential distribution between $-\delta \mathrm{V}$ (purple) and $0 \mathrm{~V}$ (white) for an interrupted water layer on the surface of a $\mathrm{C}$ thin film (purple). (For interpretation of the references to color in this figure legend, the reader is referred to the web version of the article.)

404 lowered by more than $200 \mathrm{~K}$. Taking into account the relatively high temperature ( $\geq 300 \mathrm{~K})$, we ${ }_{405}$ assume that only a small fraction of a monolayer is adsorbed on the thin films if they are kept in 406 the microscope overnight.

It is emphasized that, although the central area is charged negatively, the thin film as a whole is still a charge neutral entity. ESD can cause the desorption of neutral molecules or charged 
fragments from the surfaces. Charges possibly left behind on the thin-film surface by desorption of ions are however rapidly compensated by a current from ground, if the film has sufficient electrical conductivity and no non-conducting surface barrier; a necessity which is supposedly given for the investigated thin films.

\subsection{Electrostatic potential simulations}

Water adsorbed on a surface can be interpreted as a surface dipole layer with the positive charge pointing to the vacuum [41] which represents a potential step $\delta \mathrm{V}=\mathrm{p} /\left(\mathrm{A} \epsilon_{0}\right)$ where $\mathrm{p}$ is the dipole moment of a water molecule, and 1/A is the areal number density of water molecules on the surface. This potential step effectively changes the WF of the material [41]. If water is desorbed by ESD in the area illuminated by the PEs, this surface dipole layer is interrupted leading to a disturbance of the previously homogeneous electrostatic potential distribution on the thin-film surface. To assess the resulting change of the charge distribution and the impact on the phase shift induced on electrons propagating through the potential, we first analytically calculate the potential distribution of two interrupted dipole layers in empty space, e.g. without $\mathrm{C}$ thin film using elliptic integrals under the assumption that the field from one layer does not influence the other. This and the following calculations are conducted with radial symmetry and the central, interrupted, area in Figure 8 is actually a circular region. The result obtained without $C$ thin film is depicted in Figure $8 \mathrm{c}$ with the potential ranging from $-\delta \mathrm{V}$ (purple) to $0 \mathrm{~V}$ (white). Here, the negative potential is limited to the area between the dipole layers. The phase shift induced on a primary electron passing the circular, interrupted, area $(q=0)$ is calculated by integration of the potential along the beam path and results in $-\mathrm{C}_{\mathrm{E}} \mathrm{t} \delta \mathrm{V}$. This phase shift is identical to the phase shift induced on a scattered electron passing the 'wet' area far away from the center $(q>0)$ due to the potential between the two dipole layers resulting in a net phase shift of 0 .

If a $\mathrm{C}$ thin film, for now assumed to be a metal, is put in between the two interrupted dipole layers, the electrons in the conduction band will 'see' the potential distribution caused by the water dipoles. The electrons in the central, 'dry', part, where the water was removed by ESD, will have a lower energy than the electrons in the surrounding, 'wet', areas. If the radius of the illuminated patch $\mathrm{R}$ is much larger than the film thickness, the potential difference within the thin film will amount to $\delta \mathrm{V}$. The electrons close to the surface in the wet areas of the thin film will then drift to the dry patch and lead to a local negative charging of the dry patch until the potential in the $\mathrm{C}$ thin film is in an equilibrium state. Figure $8 \mathrm{~d}$ shows the $\mathrm{C}$ thin film, the interrupted water layer on the surfaces and the resulting potential distribution. The exact distribution of the negative charging is not accessible analytically and is only indicated in the $\mathrm{C}$ thin film by the markers ( + and - in Figure 8d). The redistribution of charges causes a potential of $-\delta \mathrm{V}$ (purple) in the $C$ thin film and in the area just above the dry patch while the potential directly above the dipole layer is $0 \mathrm{~V}$ (white). This situation is identical to assuming a thin film with a circular patch at a negative potential $-\delta \mathrm{V}$ in the illuminated area.

To reveal the dependence of the phase shift on the amount of WF change, calculations of the electrostatic potential distribution in the vacuum area above a charged thin film were conducted. The illuminated thin film is modeled as a surface with a circular (radius R) unit-step potential variation $\delta \mathrm{V}$ corresponding to the expected change of the WF. The solution of the Laplace equation yields the potential distribution displayed in Figure $8 \mathrm{~d}$ and the integration of the potential along a vertical beam path results in the phase shift imposed on electrons propagating through the thin film. In this model, the phase shift imposed on the PEs $(q=0)$ relative to electrons scattered at very high angles $(q=\infty)$ is given by 


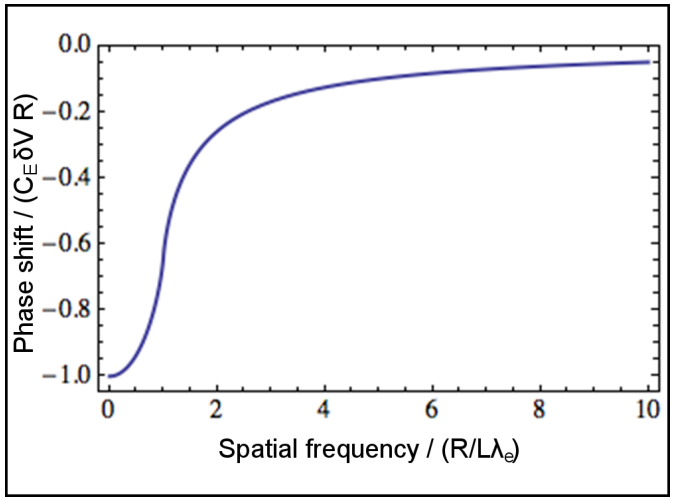

Figure 9: Generic profile of the phase shift imposed on a fast electron propagating through the potential distribution in Figure $8 \mathrm{~d}$ obtained by integration along the beam path. $\mathrm{L}$ is the camera length.

$$
\phi_{P P}=C_{E} \cdot R \cdot \delta V
$$

where the interaction constant is $C_{E}=6.53 \cdot 10^{6} \frac{1}{m \cdot V}$ for $300 \mathrm{keV}$ electrons [25]. The phase shift imposed on a PE propagating through the area with increased WF is linearly dependent on the radius of the area $\mathrm{R}$ and on the amount of $\mathrm{WF}$ change $\delta \mathrm{V}$. It is noted that the radius $\mathrm{R}$ of the area is not identical with the beam size which will be discussed in the following section. As the WF outside of the circular patch is reduced by the adsorbed water layer, $\delta \mathrm{V}$ is negative which signifies a negative phase shift.

Figure 9 shows the generic phase profile in dependence of the spatial frequency $\left(\mathrm{R} /\left(\lambda_{e} \mathrm{~L}\right)\right)$ obtained by integration of the potential distribution with $\mathrm{L}$ being the camera length. The phase profile initially exhibits a steep gradient before evolving into a long flat tail. The top-hat potential assumed in the simulations thus does not result in a top-hat phase shift profile. The long tail implies that even if the patch with changed WF is very small, the electrostatic potential can reach far out and can still affect high spatial frequencies which can complicate the experimental determination of $\phi_{P P}$. In practical experiments the shallow gradient above spatial frequencies of $2 \mathrm{R} /\left(\lambda_{e} \mathrm{~L}\right)$ will be difficult to detect due to the interplay with the defocus and can possibly be interpreted as a boundary of the phase shift tails.

So far, we assumed the $\mathrm{C}$ thin films to have metal-like character signifying highly mobile charge carriers within the thin film. For a transition into semi-conducting properties, the charge carrier density and mobility in the thin film will decrease and a space-charge region (SCR) at the interface between wet and dry patch will form. The SCR is infinitesimally small in case of a metal but can be large for semiconductors or insulators. However, as long as the width of the SCR is much smaller than the radius $\mathrm{R}$ of the illuminated patch, the total amount of phase shift induced on the primary electrons with respect to electrons scattered to high spatial frequencies remains unchanged. Equation 3 is still valid and only the shape of the phase profile (Figure 9) is affected, i.e., the profile will extend to higher spatial frequencies. As the investigated aC thin films only showed minor charging effects, it is reasonable to assume that their properties are close to a metal-like behavior at the investigated temperatures.

With equation 3, we can compare experimental results with the simulations and estimate a value for $\delta \mathrm{V}$. In case of the SLG thin film, the spatial frequencies affected by the charged area and 
the resulting phase shift profile can be estimated by an increasing shift of Thon rings (Figure 5a) to be in the order of $0.02 \mathrm{~nm}^{-1}(\approx 250 \mathrm{~nm})$. This size can be linked to the boundary $2 \mathrm{R} /\left(\lambda_{e} \mathrm{~L}\right)$. Under the assumption, that both, upper and lower, thin-film surfaces are equally charged, the observed phase shift of $-0.2 \pi$ has to be divided by a factor of $2 . \delta \mathrm{V}$ can then be calculated to be $-0.4 \mathrm{~V}$ for the SLG thin film at RT which corresponds well to expectations from literature [40].

By taking the dipole moment of an isolated water molecule $\mathrm{p}_{\mathrm{H}_{2} \mathrm{O}}=6.18 \cdot 10^{-30} \mathrm{C} \cdot \mathrm{m}$ one can estimate the upper limit of the potential step $\delta \mathrm{V}$ caused by a monolayer of adsorbed water on the surface. With the area $A=0.18 \mathrm{~nm}^{2}$ occupied by a single water molecule in case of full coverage [41] and the vacuum permittivity $\epsilon_{0}=8.85 \cdot 10^{-12} \mathrm{~F} \cdot \mathrm{m}^{-1}$, the potential step results in $4 \mathrm{~V}$. This estimation is an upper limit to $\delta \mathrm{V}$, while in reality the work function decrease is expected to be substantially lower due to three aspects: 1 . The dipole moment of chemisorbed water molecules will decrease compared to an isolated water molecule due to electronic structure relaxation and charge transfer with the $\mathrm{C}$ atoms of the surface; 2 . We expect the coverage of the surface to be less than a full monolayer at the studied temperatures and 3. the thin films exhibit a surface roughness which can lead to a loss of dipole moment due to a misalignment of the water molecules with respect to the thin-film surface.

We assumed that water is the main component of the adsorbed surface layer and that water reduces the $\mathrm{WF}$ of the investigated $\mathrm{C}$ thin films. However, it is possible that impurities in the adsorbed water layer or on the film surfaces, such as the presence of metal ions, can change the polarity of the charging or affect the electrical properties such as the WF of the film. As we used double-distilled water for preparation of the thin films, we assume that such impurities are present only in a negligible amount. It is noted, however, that different adsorbed molecules will as well influence the WF of the thin film if their adsorption is linked with a charge transfer between molecule and thin-film atoms. Such a charge transfer will cause a redistribution of charges on the thin-film surfaces. If the adsorbed molecules are removed by ESD in a small area, it will again result in a charging of the thin film in the illuminated area. The presented model is thus also valid for different adsorbates which can be desorbed by ESD and lower the WF.

The removal of water in the dry area and the resulting potential distribution not only causes a negative phase shift on transmitting electrons but additionally has an effect on the detectable SE signal. A generated SE can only escape from the thin film if its kinetic energy is larger than the WF. As the WF is restored to the nominal value in the dry area, the energy barrier for a SE to escape from the thin film increases, which leads to a decreasing number of detectable SEs. This effect is indeed observed in Figure 4, where the SE signal is plotted as a function of the areal dose for the different thin films. The SE signal is almost constant for thin films not showing a charging effect such as the SLG thin film at $450{ }^{\circ} \mathrm{C}$ (supplementary data). Only a minor initial decrease of the SE signal is observed for the Thread aC at $300{ }^{\circ} \mathrm{C}$ where charging also was below the measurable level $(<\approx 0.02 \pi)$. On the other hand, a decreasing SE signal is observed for charging thin films such as the PVD aC thin film at RT (Figure 4a). The SLG thin film at RT which shows charging effects seems to have a stable SE signal but a closer look reveals a faint decrease at higher areal doses (supplementary data). Additionally, the SE signal is only acquired up to an areal dose of $600 \mathrm{C} / \mathrm{cm}^{2}$ while the measured phase shift appeared at higher doses $\left(1000 \mathrm{C} / \mathrm{cm}^{2}\right)$ indicating a possible further decreasing SE signal at doses $>600 \mathrm{C} / \mathrm{cm}^{2}$. Overall, the measured SE traces are consistent with the model of an increasing WF in the illuminated area and a corresponding suppression of escaping SEs.

The discussed model is also consistent with the $t / \lambda$ measurements (Figure 2 ). The desorption of a thin layer of adsorbed $\mathrm{H}_{2} \mathrm{O}$ molecules only has an insignificant effect on the overall $t / \lambda$ value of an $\mathrm{aC}$ thin film resulting in the flat evolution. A measurable decrease of $t / \lambda$ is only 
observed when the desorbed molecules represent a substantial content of the thin film such as for the SLG thin film at RT or the EB aC thin film on the first day. In those cases, the type of molecule desorbing from the thin film is most likely of hydrocarbon nature possibly containing considerable amounts of water.

\subsection{Experimental influences on the charging behavior}

The simulations show that the charging model based on an ESD induced change of the WF in the illuminated area is well compatible with the experimental data. According to the simulations, the strength of the charging effect is mainly influenced by the radius $\mathrm{R}$ of the charged area and the amount of the induced WF change $\delta \mathrm{V}$. In this section we assess the main parameters that influence both $\mathrm{R}$ and $\delta \mathrm{V}$ in a particular experiment such as, e.g., the thin-film temperature and the beam current density.

$\delta \mathrm{V}$ depends on the electrical and structural properties of the thin film and the water coverage of the surfaces. The studies of the change of the WF induced by $\mathrm{H}_{2} \mathrm{O}$ adsorbed on metal surfaces showed that the WF decreases with increasing water coverage and saturates once a coverage with one monolayer is reached [41,43]. The implication for our experiments is that the amount of water adsorbed on the thin-film surfaces has direct influence on $\delta \mathrm{V}$. The lower the number of adsorbed $\mathrm{H}_{2} \mathrm{O}$ molecules on the thin-film surfaces, which is determined by the thin-film temperature and structure as well as the partial pressure of water in the residual gas atmosphere, the lower is the observed charging effect. A higher temperature increases the available thermal energy causing more water to desorb and vice versa. Ad- and desorption, however, are relatively slow processes so that a fast change of the thin-film temperature does not immediately change the number of adsorbates on the thin-film surfaces. The adsorption of molecules is also influenced by the morphology of the thin film. A film with high surface roughness or film porosity provides more adsorption sites than a homogeneous thin film meaning that, under identical experimental conditions, the coverage with water can vary between different aC thin films.

The residual gas composition in the Hitachi HF-3300 mainly contains water (supplementary data). The higher the partial pressure of water, the higher is the number of adsorbed $\mathrm{H}_{2} \mathrm{O}$ molecules on the thin-film surfaces. The vacuum in a transmission electron microscope is not ultra-high and typically in the range of a few $10^{-7}$ mbar. As the HF-3300 microscope is baked regularly over the weekends, the vacuum stays at values below $5 \cdot 10^{-8}$ mbar and its vacuum is supposedly better than in a typical transmission electron microscope. This implies that the amount of adsorbed water and thus the corresponding charging effects are less pronounced in the HF-3300.

It is emphasized, that the area on the thin film where the WF change is induced by the illuminating electrons $(\mathrm{R})$ does not have sharp borders but is expected to be diffuse. The ESD of water molecules from the surface layer is generated by SEs with a specific spatial distribution which in general is much broader than the PE beam diameter. Water molecules are therefore not only desorbed in the center of the illuminated area but in an area with the size of the mean-free path of the SEs in the $\mathrm{aC}$ thin film. The edges of this area are only reached by a small number of SEs leading to a strong reduction of the ESD rate at larger distances from the beam center. While the water molecules within the illuminated area are desorbed quickly, further illumination with the electron beam will cause an enlargement of the patch where the WF is increased. Longer illumination times should then result in a slowly increasing radius $\mathrm{R}$ and observed negative phase shift $\phi_{P P}$. The diffuse edges of the dry patch will only influence the phase shift profile (Figure 9) but not the final amount of $\phi_{P P}$.

The beam current density has a major influence on the charging behavior. The beam current itself is suspected to only have a minor influence on the charging effects if it stays within typical 


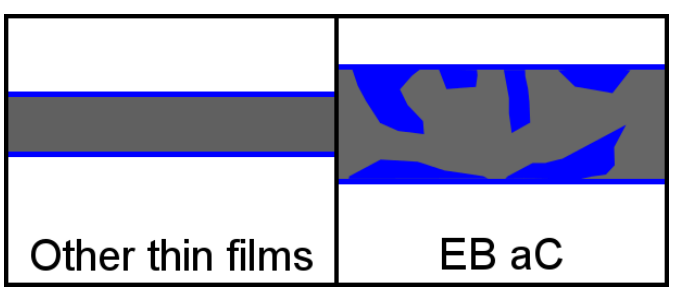

Figure 10: Schematic drawing of the assumed structure for (a) the Thread aC, PVD aC and SLG thin films and (b) the $\mathrm{EB}$ aC thin film. The thin films are depicted with a gray color and the blue color indicates adsorbed molecules. (For interpretation of the references to color in this figure legend, the reader is referred to the web version of the article.)

values for STEM and if the beam diameter on the thin film is kept constant. A lower beam current then will of course lower the rate at which ESD takes place but it is suspected to not have an influence on the final amount of observable phase shift. The time scale at which water will re-adsorb on the thin-film surface is drastically higher than typical STEM measurement times considering the low partial pressure of water within the microscope. Once all water molecules are desorbed during PE illumination, a steady state is reached. If the beam is switched off, water molecules will start to re-adsorb on the illuminated area and possibly diffuse along the sample surface. However, both processes are assumed to happen in a time scale of minutes and hours which can explain the fact that HFPP patches remain charged for a longer period of time [14, 31]. We did not study the longevity of the charged patch in the presented experiments and can thus only speculate about the speed of the re-coverage of the surface with water.

In contrast to the beam current, a significant influence on the detectable phase shift is given by the beam diameter. A change in the size of the area in which the WF changes will directly influence the radius $\mathrm{R}$ (Equation 3 ) and thus increase the phase shift induced on transmitting electrons. Here, the beam diameter was small $(\approx 20 \mathrm{~nm})$ compared to the charged area. If the beam diameter is however increased strongly much larger phase shifts could be be achieved.

\subsection{Charging of the EB aC thin film}

To understand the observed differences between Thread and PVD aC compared to the EB $\mathrm{aC}$ thin film which still charges even if it is extensively heated to $260{ }^{\circ} \mathrm{C}$ in the microscope (Figure 6b), the structure of the thin films has to be analyzed. The EB aC shows a strong decrease in thickness when illuminated shortly after insertion in the microscope and subsequent heating to $260^{\circ} \mathrm{C}$. $t / \lambda$ decreases from $\approx 0.2$ by a factor of 2 before starting to rise again (Figure $2 b$ ). This behavior can be explained if a porous structure of the $\mathrm{EB} \mathrm{aC}$ thin film is assumed. Without a cleaning step, the $\mathrm{EB} \mathrm{aC}$ thin film surface and its pores are covered with hydrocarbon molecules (Figure 10b). The initial value for $t / \lambda$ therefore includes both the thickness of the actual $\mathrm{EB}$ aC thin film and of the hydrocarbon molecules. Previous measurements showed that heating of an aC thin film to $275{ }^{\circ} \mathrm{C}$ overnight leads to a desorption of the hydrocarbon molecules [16]. The value for $t / \lambda$ observed on the second day after overnight heating to $260^{\circ} \mathrm{C}$ then reflects the actual thickness of the $\mathrm{EB} \mathrm{aC}$ thin film. This implies that initially, about half of the measured thickness originated from adsorbed hydrocarbon molecules. Assuming that the thickness of an adsorbed hydrocarbon layer is self-limited to a few monolayers, the observed $t / \lambda$ composition can only be explained by assumption of a porous thin film structure as schematically depicted in Figure 10b.

This assumption is confirmed by the comparative XPS measurements of the PVD and EB $\mathrm{aC}$ thin films (Table 2). XPS is a technique sensitive to the first few $\mathrm{nm}$ of a sample only. 
XPS measurements on the $\mathrm{aC}$ thin films should reflect the fraction of $\mathrm{sp}^{2}$-hybridized $\mathrm{C}$ atoms which was determined by EELS measurements to be $\approx 70 \%$ independent of the type of aC thin film [16]. However, the binding energy of $\mathrm{sp}^{3}$-hybridized $\mathrm{C}-\mathrm{C}$ bonds is identical to $\mathrm{C}-\mathrm{H}$ bonds meaning that $\mathrm{C}-\mathrm{H}$ bonds present in hydrocarbon molecules adsorbed on the thin film can affect the determination of the $\mathrm{sp}^{2}$ to $\mathrm{sp}^{3}$ ratio. The XPS measurement performed on the as-prepared $\mathrm{PVD}$ aC thin film reveals the presence of $\approx 70 \% \mathrm{sp}^{2}$-hybridized C-C bonds and about $10 \%$ of the total $\mathrm{C}$ atoms are bonded with $\mathrm{O}$ which can be attributed to surface contamination (c.f. Table 2). After the Ar sputter cleaning procedure, the $\mathrm{sp}^{2}$ fraction slightly increases to $75 \%$ due to the removal of contaminants and the amount of $\mathrm{O}$ bonds is strongly decreased. The experimental results obtained on the PVD aC thin film are well compatible with the results expected for a homogeneous thin film with low roughness and a thin adsorbed contamination layer.

A different situation is found for the $\mathrm{EB}$ aC thin film. The high percentages of $\mathrm{C}-\mathrm{O}$ and $\mathrm{C}-\mathrm{H}$ bonds for the uncleaned thin film are attributed to the presence of large amounts of contamination which almost completely cover the actual thin film. Ar-sputtering removes a substantial part of the contamination but the amount of $\mathrm{C}$ bonds with $\mathrm{O}(9 \%)$ is still significantly higher compared to the PVD aC thin film (4.5\%). This implies that contamination is also present within the actual $\mathrm{EB}$ aC thin film indicating a high surface roughness and a porous structure. Additionally, the absence of $\mathrm{C} \pi-\pi^{*}$ bonds in the $\mathrm{EB}$ aC thin film suggests that graphitic planes are not present in the thin film.

The structural differences between the $\mathrm{EB}$ aC and all the other $\mathrm{C}$ thin films and its significance for the charging effects can be summarized as follows: The PVD aC, Thread aC and SLG thin films are homogeneous and have a very low surface roughness meaning that a limited number of sites are available for molecules to adsorb. In contrast, the high roughness and porosity of the $\mathrm{EB}$ aC thin film results in a significantly higher active surface area. Effects which are related to adsorbed surface layers are therefore suspected to be much more pronounced for the $\mathrm{EB}$ aC compared to the other $\mathrm{C}$ thin films.

The rapid decrease in thickness of the $\mathrm{EB}$ aC thin film during electron-beam illumination shortly after insertion and heating to $260{ }^{\circ} \mathrm{C}$ is recognized as electron-stimulated desorption (ESD) of hydrocarbon molecules. As hydrocarbon molecules desorb overnight at a temperature of $260{ }^{\circ} \mathrm{C}$, the thermal energy available $(\approx 45 \mathrm{meV})$ is sufficient to initiate the desorption processes. However, the desorption rate is limited and it takes a long time for the contamination to desorb. When the thin film is illuminated shortly after heating to $260{ }^{\circ} \mathrm{C}$, the time at elevated temperature was not sufficient for all molecules to desorb. The focused electron-beam illumination then stimulates the desorption of the remaining adsorbed molecules in the illuminated area leading to the observed rapid decrease in thickness. In parallel with ESD, but at a much slower rate, the deposition of a contamination layer takes place [16]. The deposition of contamination becomes dominant once all molecules in the illuminated area, including the water molecules chemisorbed on the surface, are desorbed, i.e. there is no more material to be removed. Contamination may still be present as mobile hydrocarbon molecules can diffuse along the film surface to the irradiated area $[16,32]$. After the desorption ceases, surface sites of the carbon thin film itself are available for hydrocarbon molecules to bond and to form a contamination layer leading to the observed thickness increase. This interplay between desorption and deposition can also be observed in the analysis of the phase shift evolution of the Volta PP at $60{ }^{\circ} \mathrm{C}$ (Figure 7a, [14]). The evolution first shows a negative phase shift caused by ESD followed by a trend to positive phase shifts induced by contamination. A detailed description of the involved physical processes is not possible solely from the presented experiments and so several parameters remain unclear, e.g., the ratio between desorption and deposition or the mixture of molecules present on the thin 
film.

The time resolution of the PCTF measurements is not sufficient to unambiguously resolve the phase shift evolution of the EB aC directly after insertion (Figure 6a). The initial phase shift step has a value close to $\pi / 2$ which can be interpreted as a positive or negative phase shift. As the following phase shift steps are smaller than $\pi / 2$ and tend toward a negative phase shift, the first guess is that the initial phase shift step is as well negative, which will be denoted as 'negative interpretation' in the following. The observed thickness change (Figure 2b) can be used to estimate an expected phase shift if values for the MIP (here $9 \mathrm{~V})$ and the inelastic MFP $(150 \mathrm{~nm})$ are assumed (Figure 6a). A comparison with the negative interpretation shows that the measured phase shift is about $\pi$ larger than expected from the thickness decrease (c.f. Figure $2 b$ and $6 a$ ). In addition to the decreasing thickness, a negative charging must occur to explain the higher negative phase shift. This negative charging could be explained by ESD and an accompanying WF change. As hydrocarbon molecules are only physiosorbed on the thin-film surface, a strong influence on the WF is not expected and the major part of the WF change is assumed to originate from ESD of water.

The phase shift evolution on the first day can also be interpreted as an initial positive phase shift step. This positive phase shift could be caused by a positive charging of the irradiated area, e.g., by positive charge trapping in the insulating hydrocarbon layer. As the adsorbed hydrocarbon molecules are rapidly desorbed during further illumination, the positive phase shift is present for only a few seconds and measurement points. After desorption of the adsorbed hydrocarbon molecules, the phase shift evolution for an initial positive phase shift step follows the expected evolution from the $t / \lambda$ measurements (Figure $6 a$ ) indicating a lack of charges in the illuminated area.

The presence of a large amount of hydrocarbon molecules on the $\mathrm{EB}$ aC thin film shortly after insertion and heating of the thin film complicates the interpretation of the experimental results and we can only speculate on the involved processes. Both, positive and negative, initial phase shift possibilities can be explained by either negative or positive charging of the illuminated area. The data acquired after two and four days of consecutive heating of the thin film however clearly show the effect of the different structure of the $\mathrm{EB}$ aC compared to the other thin films. After one day of heating to $260^{\circ} \mathrm{C}$, most hydrocarbon molecules are desorbed from the thin-film surface and the obseryed negative phase shift of $\approx-0.15 \pi$ can be explained by ESD of water and an accompanying WF change. The amount of water adsorbed on the thin film is suspected to be higher compared to the other thin films due to the structural differences such as increased surface roughness which leads to the stronger charging of the $\mathrm{EB} \mathrm{aC}$ thin film. The amount of measured phase shift only slightly decreased from the second to fourth day of consecutive heating in the microscope indicating that an equilibrium state between the number of adsorbed $\mathrm{H}_{2} \mathrm{O}$ molecules on the thin-film surfaces and the partial pressure of water in the residual gas atmosphere is established. The negative charging can also be observed in the SE signal. The EB aC thin film exhibits a decreasing SE signal caused by the increased WF and the drop is decreasing with the time kept in the microscope (Figure $4 \mathrm{~b}$ ) corresponding well to the observed decreasing induced phase shift (Figure 6b).

\subsection{Implications for HFPP imaging and related charging phenomena}

The local change of the WF of a thin film induced by ESD of surface layers can affect numerous applications in EM. The effect causes a more subtle type of charging with induced phase shifts below $\pi$ which nevertheless can hamper a correct analysis of acquired data. Here we dis- 
cuss several possible applications, where ESD and an accompanying change of the WF can play a significant role.

The described charging phenomenon is a plausible explanation for the functionality of the HFPP $[8,31]$ and its implementations including the Volta PP [14]. The aC thin film used as Volta PP in [14] and as HFPP in [31] was fabricated using the identical device type as the EB aC thin film analyzed in this work. The Volta PP is used at a temperature of $225^{\circ} \mathrm{C}$ which is slightly lower than the EB aC thin-film and HFPP temperature of $260{ }^{\circ} \mathrm{C}$ and the beam diameters were 1 $\mu \mathrm{m}, 20 \mathrm{~nm}$ and $100 \mathrm{~nm}$, respectively. The thin films show negative charging upon electron-beam illumination with the reported phase shift being about four times higher for the Volta PP than the results presented here (c.f. Figures $6 \mathrm{~b}$ and 7a). An ESD-induced change of the WF is a plausible explanation for the functionality of the negatively charged PPs. Several factors lead to the higher negative PS of the Volta PP: Firstly, the larger beam diameter on the Volta PP causes a larger patch radius $\mathrm{R}$ yielding a higher phase shift. Secondly, it is reasonable to assume that the vacuum in the regularly baked Hitachi HF-3300 is better than in the cryo microscopes used in [14, 31] where water supposedly has a higher partial pressure. Thirdly, together with the lower operating temperature of the Volta PP the higher partial pressure of water leads to more water molecules adsorbed on the thin-film surface leading to a higher change in WF due to ESD. Danev et al. [14] claimed a Volta potential induced by the illuminating electron beam to be the explanation for the functionality of their experimental observations. Our findings and the established ESD model now give the physical explanation for the origin of the change in potential. ESD of water molecules from the surface leads to a local change in the WF of the aC thin film which can be interpreted as a local negative charge or potential inducing the observed PS.

Figure 11 gives a graphical overview over the possible implementations of a HFPP which all have in common that an electron-beam induced process causes an additional phase shift of the PEs. Hettler et al. [16] reported that contamination deposited by an electron beam with high current density can be used as a HFPP if the deposition process can be controlled. This control, however, is difficult to achieve and has not been applied yet. The first implementation of a HFPP by Malac et al. [8] was explained by positive charges in insulating layers left behind by escaping SEs [44]. There are further possible implementations of HFPP concepts, especially if material other than $\mathrm{aC}$ is utilized. Any electron-beam induced process which is separating charges perpendicular to the electron beam can be a possible explanation. Structural changes of a material induced by electrons can, e.g., change the MIP of the thin film and lead to the desired phase shift. Additionally, local heating could be used to induce diffusion of specific elements in alloys or to induce electric fields in thermoelectric materials.

Our findings have several implications on the applicability of the negative-charging variation of the HFPP. As the EB aC has a high surface roughness, local structure variations are highly probable. The PS resulting from focused electron-beam illumination due to ESD and the accompanying WF change will depend on the local structure due to different amounts of adsorbed water and local variations of the electronic properties. The established PS is therefore not stable and varies with the positioning of the illuminating electron beam on the $\mathrm{aC}$ thin film. This is reflected by the strongly varying performances of the used HFPPs [31]. An additional observation for the Volta PP is that the induced PS does not reach a steady state but further increases with illumination time (Figure 7a). This can be explained by a further increasing patch size $\mathrm{R}$ with increased WF due to ESD of water by secondary electrons and electrons scattered to low spatial frequencies in the specimen. The scattered electrons have a strongly reduced intensity compared to the unscattered electrons yielding two or more different time constants.

In contrast to our experimental results, Brink et al. [2] claimed a positive charging of the in- 


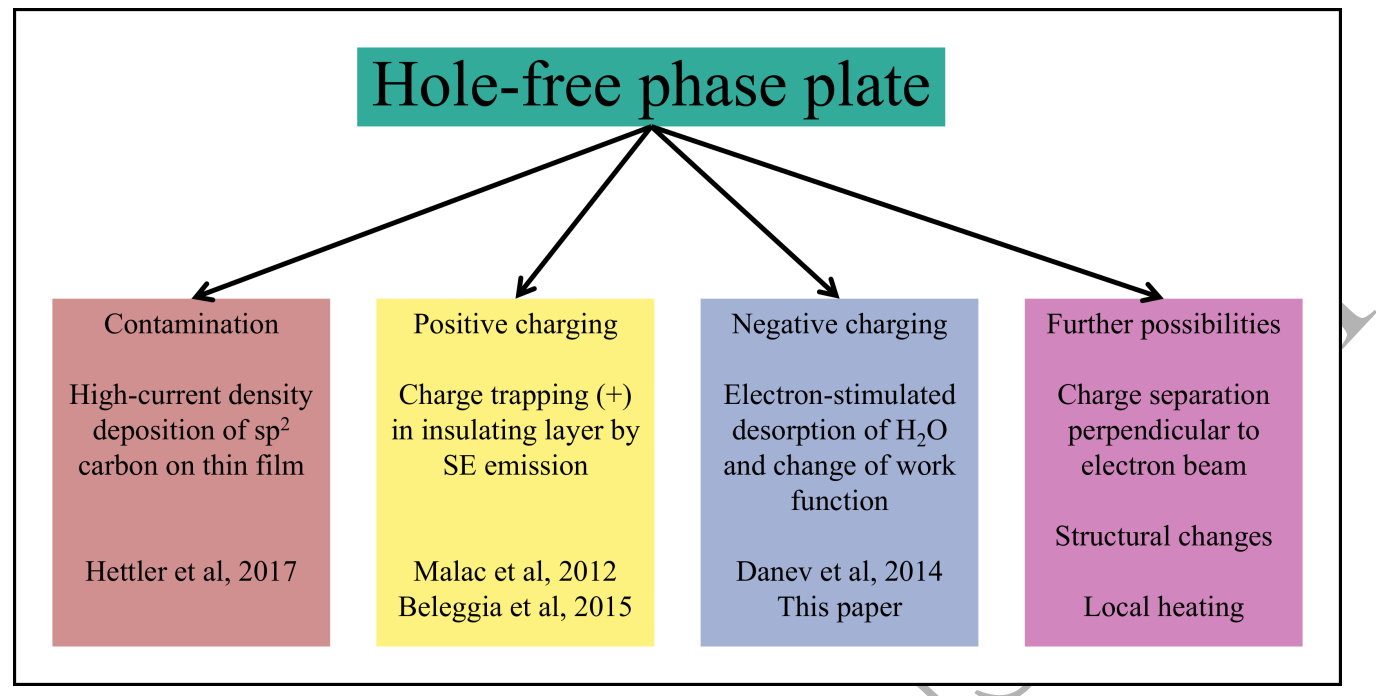

Figure 11: Graphical illustration of possible HFPP implementations.

vestigated $\mathrm{aC}$ thin film. The origin was supposed to come from highly localized positive charges in a non-conductive layer of the thin film which could not be compensated by electrons from the thin film itself due to a barrier. The experiments were conducted at the temperature of liquid nitrogen suggesting a strongly reduced conductivity of the aC thin film and a thick layer of adsorbed water on the surface. The experimental conditions differ largely from ours so that both phenomena do not contradict each other.

The findings are also important for any phase-related (S)TEM imaging. The phase induced by a thin film or a standard TEM sample is influenced by surface layers which we showed are suspect to change upon focused electron-beam illumination. Phase measurements acquired by, e.g., electron holography or focal-series reconstruction can be impaired by the described effects if the investigated area has been previously illuminated inhomogeneously with a focused electron beam. The effect of an ESD-induced change of the work function and sample charging is especially significant for SE measurements both in the SEM and TEM. The experimental results show a strong influence of the WF on the number of detectable SEs (Figure 4) if a surface layer is present. Although sample contamination is probably the most frequent artifact observed in SEM, the change of the WF and a suppression of SE emission induced by ESD of surface layers can additionally play a role in contrast formation in SEM imaging.

\section{Conclusions}

We analyzed the charging of $\mathrm{C}$ thin films under focused electron-beam illumination in a transmission electron microscope. Four different $\mathrm{C}$ thin films were studied by electron energyloss spectrosopy, hole-free phase plate (HFPP) imaging, detection of SEs and x-ray photoelectron spectroscopy to identify the physical reason for the observed charging effects. Contamination was avoided by suitable pretreatments as described in [16] which allows a clear separation of contamination and charging. The following conclusions can be drawn: 
- The investigated $\mathrm{C}$ thin films exhibit an electrical conductivity which is sufficient to compensate all charges created by illuminating high-energy electrons within the thin film if they are kept at room temperature or above. If they are free from adsorbed surface layers, the thin films do not charge.

- Negative charging is observed under intense focused electron-beam illumination if contamination due to hydrocarbon molecules is avoided by appropriate pretreatments. Suitable measures are, e.g., UV cleaning, beam showering or heating which either immobilize or remove the hydrocarbon molecules.

- Negative charging on contamination-free surfaces induces phase shifts with an amount less than $\pi / 2$. The physical reason for the negative charging is identified as electron-stimulated desorption (ESD) of adsorbed water molecules in the illuminated area which results in a local increase of the work function. The adsorbed water layer can be interpreted as a dipole layer with the positive $(\mathrm{H})$ end pointing to vacuum.

- A calculation of the electrostatic potential above a thin film with a circular patch at a negative potential yields an expression for the relative phase shift between unscattered electrons and electrons scattered to high spatial frequencies which is linearly dependent on the radius $\mathrm{R}$ of the charged area and the amount of the work function change $\mathrm{e} \delta \mathrm{V}$.

- By comparison of experiment and calculation, the amount of work function change is estimated to be $\mathrm{e} \delta \mathrm{V}=0.4 \mathrm{eV}$ in case of the single-layer graphene (SLG) thin film at room temperature.

- Heating of the $\mathrm{C}$ thin films leads to desorption of all adsorbed molecules and to a vanishing negative charging. The temperature above which the surface layers are completely desorbed depends on the structure of the thin film and was between $275{ }^{\circ} \mathrm{C}(\mathrm{PVD}$ and Thread $\mathrm{aC}$ ) and $450^{\circ} \mathrm{C}$ (SLG) for the analyzed C thin films.

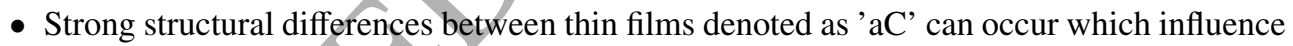
the characteristics of the negative charging.

- The functionality of the HFPP [8] and the Volta phase plate [14] can be well explained by the proposed model for the negative charging. The charging effect however depends on numerous parameters, e.g. the amount of adsorbed water on, or the local structure of the thin film, which are difficult to control. Depending on the operating parameters of the HFPP, positive charge trapping [8] and carbon contamination [16] remain plausible alternative explanations.

- Under certain conditions, ESD of hydrocarbon molecules and the deposition of hydrocarbon contamination layers can occur in parallel. The experimental results indicate that ESD is taking place much faster than the deposition of contamination.

- The described model of charging could affect any phase-related technique in (scanning) transmission electron microscopy and can influence the contrast of secondary electron images. 


\section{Acknowledgments}

S. Hettler acknowledges funding from the Karlsruhe House of Young Scientists (KHYS). The work performed at KIT was funded by the German Research Foundation (DFG). E. Kano acknowledges funding from JSPS KAKENHI Grant Number 15J04118. The support from Dr. M. Takeguchi and Dr. A. Hashimoto (NIMS, Japan) and the SLG sample offer from Dr. S. Sakai (QST, Japan) is gratefully acknowledged. The work at NINT was supported by the National Research Council of Canada. The ongoing support of Hitachi High Technologies (Naka, Japan) especially Dr. Y. Taniguchi and Hitachi High Technologies Canada (D. Hoyle and C. Soong) was crucial in developing a custom experimental setup of the Hitachi HF-3300. Collaboration with JEOL Ltd. and JEOL USA Inc. allowed us to pursue phase-plate development at NINT. In particular the help of Dr. M. Kawasaki (JEOL USA Inc.) and Dr. Y. Okura, Dr. S. Motøki, Dr. I. Ishikawa and Dr. Y. Konyuba (JEOL Ltd.) has been crucial in building the phase plate imaging at NINT. The fabrication of the EB aC thin film by Mike Marko (Wadsworth Centre, New York State University, Albany, NY, USA) and of the Thread aC thin film by Peter Hermann (LEM, Karlsruhe Institute of Technology, Karlsruhe, Germany) is acknowledged.

\section{References}

[1] R. Henderson, R. M. Glaeser, Quantitative analysis of image contrast in electron micrographs of beam-sensitive crystals, Ultramicroscopy 16 (1985) 139-150. doi : 10.1016/0304-3991 (85) 90069-5.

[2] J. Brink, M. B. Sherman, J. Berriman, W. Chiu, Evaluation of charging on macromolecules in electron cryomicroscopy, Ultramicroscopy 72 (1998) 41-52. doi : 10.1016/S0304-3991 (97) 00126-5.

[3] J. A. Berriman, P. B. Rosenthal, Paraxial charge compensator for electron cryomicroscopy, Ultramicroscopy 116 (2012) 106-114. doi:10.1016/j.ultramic.2012.03.006.

[4] K. Sader, M. Stopps, L. J. Calder, P. B. Rosenthal, Cryomicroscopy of radiation sensitive specimens on unmodified graphene sheets: reduction of electron-optical effects of charging, J Struct Biol 183 (3) (2013) 531-536. doi: $10.1016 / j \cdot j$ jb. 2013.04.014.

[5] C. J. Russo, L. A. Passmore, Progress towards an optimal specimen support for electron cryomicroscopy, Curr Opin Struc Biol 37 (2016) 81-89. doi : 10.1016/j.sbi.2015.12.007.

[6] R. F. Egerton, P. Li, M. Malac, Radiation damage in the TEM and SEM, Micron 35 (6) (2004) 399-409. doi: 10.1016/j.micron.2004.02.003.

[7] J. Cazaux, From the physics of secondary electron emission to image contrasts in scanning electron microscopy, J Electron Microsc 61 (5) (2012) 261-284. doi : 10.1093/jmicro/df s048.

[8] M. Malac, M. Beleggia, M. Kawasaki, P. Li, R. F. Egerton, Convenient contrast enhancement by a hole-free phase plate, Ultramicroscopy 118 (2012) 77-89. doi:10.1016/j.ultramic. 2012.02.004.

[9] R. M. Glaeser, K. H. Downing, Specimen Charging on Thin Films with One Conducting Layer: Discussion of Physical Principles, Microsc Microanal 10 (06) (2004) 790-796. doi:10.1017/S1431927604040668.

[10] D. M. Larson, K. H. Downing, R. M. Glaeser, The surface of evaporated carbon films is an insulating, high-bandgap material, J Struct Biol 174 (2) (2011) 420-423. doi : 10.1016/j.jsb.2011.02.005.

[11] R. Danev, K. Nagayama, Transmission electron microscopy with Zernike phase plate, Ultramicroscopy 88 (4) (2001) 243-252. doi : 10.1016/S0304-3991(01)00088-2.

[12] K. Schultheiss, F. Pérez-Willard, B. Barton, D. Gerthsen, R. R. Schröder, Fabrication of a Boersch phase plate for phase contrast imaging in a transmission electron microscope, Rev Sci Instrum 77 (3) (2006) 033701. doi : 10.1063/1.2179411.

[13] R. M. Glaeser, Invited review article: Methods for imaging weak-phase objects in electron microscopy, Rev Sci Instrum 84 (11) (2013) 111101. doi: 10.1063/1.4830355.

[14] R. Danev, B. Buijsse, M. Khoshouei, J. M. Plitzko, W. Baumeister, Volta potential phase plate for in-focus phase contrast transmission electron microscopy, PNAS 111 (44) (2014) 15635-15640. doi:10.1073/pnas . 1418377111.

[15] M. Malac, S. Hettler, M. Hayashida, M. Kawasaki, Y. O. Konyuba, Y. Okura, H. Iijima, I. Ishikawa, M. Beleggia, Computer simulations analysis for determining the polarity of charge generated by high energy electron irradiation of a thin film, Micron 100 (2017) 10-22. doi:10.1016/j.micron.2017.03.015. 
[16] S. Hettler, M. Dries, P. Hermann, M. Obermair, D. Gerthsen, M. Malac, Carbon contamination in scanning transmission electron microscopy and its impact on phase-plate applications, Micron 96 (2017) 38-47. doi: $10.1016 / j$.micron.2017.02.002.

[17] M. Bergen, Malac, M.,McLeod, R. A., D. Hoyle, Y. Taniguchi, Y. Yaguchi, J. Chen, T. Yotsuji, Centralized Instrument Control for a TEM Laboratory, Microsc Microanal 19 (Suppl 2) (2013) 1394-1395. doi:10.1017/ S1431927613008969.

[18] R. F. Egerton, Electron energy-loss spectroscopy in the electron microscope, 3rd Edition, The language of science, Springer, New York, 2011.

[19] L. Reimer, H. Kohl, Transmission electron microscopy: Physics of image formation, 5th Edition, Vol. 36 of Springer series in optical sciences, Springer, New York, NY, 2009.

[20] S. Hettler, J. Wagner, M. Dries, M. Oster, C. Wacker, R. R. Schröder, D. Gerthsen, On the role of inelastic scattering in phase-plate transmission electron microscopy, Ultramicroscopy 155 (2015) 27-41. doi:10.1016/j . ultramic.2015.04.001.

[21] E. Kano, M. Takeguchi, J.-i. Fujita, A. Hashimoto, Direct observation of Pt-terminating carbyne on graphene, Carbon 80 (2014) 382-386. doi:10.1016/j. carbon.2014.08.077.

[22] C. Soong, P. Woo, D. Hoyle, Contamination Cleaning of TEM/SEM Samples with the ZONE Cleaner, Micros Today 20 (06) (2012) 44-48. doi : 10.1017/S1551929512000752.

[23] S. J. Pennycook, P. D. Nellist, Scanning transmission electron microscopy: Imaging and analysis, Springer, New York, 2011.

[24] P. Wachsmuth, R. Hambach, G. Benner, U. Kaiser, Plasmon bands in multilayer graphene, Phys. Rev. B 90 (23) (2014) 235434. doi:10.1103/PhysRevB.90.235434.

[25] E. Völkl, L. F. Allard, D. C. Joy, Introduction to electron holography, Springer, New York, 1999.

[26] M. Wanner, D. Bach, D. Gerthsen, R. Werner, B. Tesche, Electron holography of thin amorphous carbon films: measurement of the mean inner potential and a thickness-independent phase shift, Ultramicroscopy 106 (4-5) (2006) 341-345. doi:10.1016/j.ultramic.2005.10.004.

[27] M. Dries, S. Hettler, T. Schulze, W. Send, E. Müller, R. Schneider, D. Gerthsen, Y. Luo, K. Samwer, Thin-Film Phase Plates for Transmission Electron Microscopy Fabricated from Metallic Glasses, Microsc Microanal 22 (5) (2016) 955-963. doi:10.1017/S143192761601165X.

[28] F. Sette, G. K. Wertheim, Y. Ma, G. Meigs, Lifetime and screening of the C 1s photoemission in graphite, Phys Rev B 41 (14) (1990) 9766-9770. doi : 10.1103/PhysRevB.41.9766.

[29] S. T. Jackson, G. R. Nuzzo, Determining hybridization differences for amorphous carbon from the XPS C1s envelope, Appl surf sci 90 (1995) 195-203. doi : 10.1016/0169-4332(95)00079-8.

[30] H. Estrade-Szwarckopf, XPS photoemission in carbonaceous materials: A "defect" peak beside the graphitic asymmetric peak, Carbon 42 (8-9) (2004) 1713-1721. doi:10.1016/j . carbon. 2004.03.005.

[31] M. Marko, C. Hsieh, E. Leith, D. Mastronarde, S. Motoki, Practical Experience with Hole-Free Phase Plates for Cryo Electron Microscopy, Microsc Microanal 22 (6) (2016) 1316-1328. doi : 10.1017/S143192761601196X.

[32] J. J. Hren, Specimen contamination in analytical electron microscopy: Sources and solutions, Ultramicroscopy 3 (1979) 375-380. doi : 10.1016/S0304-3991 (78)80057-6.

[33] E. M. Williams, J. L. de Segovia, Electron stimulated desorption of ions from surfaces: Techniques, methodology and some recent findings with water at metals and semiconductors, Vacuum 39 (7/8) (1989) 633-642. doi: 10.1016/0042-207X (89)90006-7.

[34] R. D. Ramsier, J. T. Yates, Electron-stimulated desorption: principles and applications, Surf Sci Rep 12 (1991) 243-378. doi: 10.1016/0167-5729(91)90013-N.

[35] W. F. van Dorp, C. W. Hagen, A critical literature review of focused electron beam induced deposition, J Appl Phys 104 (8) (2008) 081301. doi: 10.1063/1.2977587.

[36] I. Utke, P. Hoffmann, J. Melngailis, Gas-assisted focused electron beam and ion beam processing and fabrication, J. Vac. Sci. Technol. B 26 (4) (2008) 1197. doi:10.1116/1.2955728.

[37] W. F. van Dorp, T. W. Hansen, J. B. Wagner, J. T. M. De Hosson, The role of electron-stimulated desorption in focused electron beam induced deposition, Beilstein J Nanotechnol 4 (2013) 474-480. doi: 10.3762/bjnano. 4.56.

[38] R. L. Wells, T. J. Fort, Adsorption of water on clean gold by measurement of work function changes, Surf Sci 32 (1972) 554-560. doi:10.1016/0039-6028(72) 90182-3.

[39] J. M. Heras, L. Viscido, Work function changes upon water contamination of metal surfaces, Appl surf sci 4 (1980) $238-241$.

[40] P. A. Thiel, T. E. Madey, The interaction of water with solid surfaces: Fundamental aspects, Surf Sci Rep 7 (1987) 211-385. doi:10.1016/0167-5729(87)90001-X.

[41] J. M. Heras, G. Estiú, L. Viscido, The interaction of water with clean palladium films: A thermal desorption and work function study, Appl surf sci 108 (455-464). doi:10.1016/S0169-4332(96)00686-1.

[42] M. A. Henderson, The interaction of water with solid surfaces: fundamental aspects revisited, Surf Sci Rep 46 
(2002) 1-308. doi:10.1016/S0167-5729(01)00020-6.

[43] J. M. Heras, G. Estiú, L. Viscido, Annealing behaviour of clean and oxygen covered polycrystalline Palladium films: a work function and electrical resistance study, Thin Solid Films 188 (1990) 165-172. doi: 10.1016/0040-6090 (90) 90202-0.

[44] M. Beleggia, M. Malac, T. Rowan, R. F. Egerton, M. Kawasaki, Y. Okura, R. A. McLeod, Electron beaminduced charging and modifications of thin films, Microsc Microanal 21 (S3) (2015) 1385-1388. doi:10.1017/ S1431927615007710. 